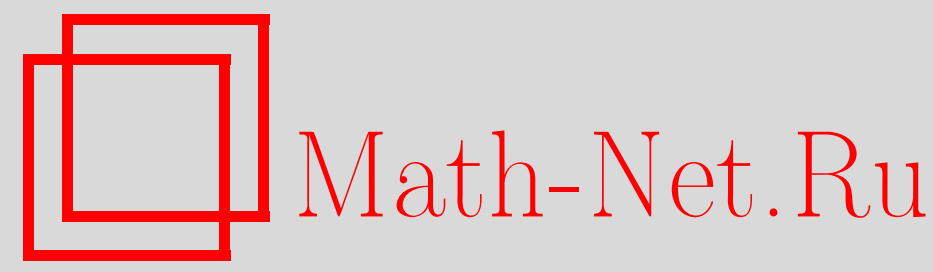

В. В. Коннов, Дифференциально-геометрический признак квадратичных вложений Веронезе, Изв. РАН. Сер. матем., 2000, том 64, выпуск 5, 21-44

DOI: https://doi.org/10.4213/im303

Использование Общероссийского математического портала Math-Net.Ru подразумевает, что вы прочитали и согласны с пользовательским соглашением http: //www.mathnet.ru/rus/agreement

Параметры загрузки:

IP : 3.95 .254 .165

26 апреля 2023 г., $15: 20: 58$ 
УДК 514.76

\author{
В. В. Коннов
}

\title{
Дифференциально-геометрический признак квадратичных вложений Веронезе
}

\begin{abstract}
Находится признак квадратичных многообразий Веронезе. Доказывается, что во множестве гладких $n$-мерных подмногообразий проективного пространства $P^{N}$ размерности $N=n(n+3) / 2$ только многообразия Веронезе обладают следующими двумя свойствами: 1) касательные проективные пространства для любой пары точек пересекаются в точке; 2) соприкасающееся проективное пространство в каждой точке совпадает с объемлющим пространством. Этот результат обобщает на случай произвольного $n$ признак двумерной поверхности Веронезе в $P^{5}$, доказанный Гриффитсом и Харрисом. Кроме этого в работе находится критерий того, что пара подмногообразий в $P^{N}$ принадлежит одному многообразию Веронезе. Приводятся вычислительные формулы, необходимые для практического использования доказанных признаков.
\end{abstract}

Библиография: 9 наименований.

\section{$\S 1$. Введение}

Многообразия Веронезе являются объектом интенсивного изучения не только алгебраической, но и дифференциальной геометрии. Так, в работе [8] вводится понятие структуры Веронезе (Veronese-structure) и находятся необходимые и достаточные условия, при выполнении которых гладкое многообразие может быть вложено в проективное пространство как квадратичное многообразие Веронезе. Ряд интересных результатов по дифференциальной геометрии многообразий Веронезе можно найти в [2], [5]-[8].

В работе [4] (см. также [1]) найден признак двумерной квадратичной поверхности Веронезе в пятимерном проективном пространстве. Он заключается в том, что, во-первых, соприкасающаяся плоскость в каждой точке поверхности имеет максимально возможсную размерность и, во-вторых, любая пара касательных плоскостей пересекается в точке. Несложно показать, что квадратичное многообразие Веронезе любой размерности обладает этими двумя свойствами. Поэтому вполне естественной выглядит гипотеза о том, что при выполнении этих двух условий гладкое подмногообразие произвольной размерности вырождается в квадратичное многообразие Веронезе. Однако, как показьвают [1], [4], уже для двумерных поверхностей доказательство этой гипотезы оказывается весьма не тривиальным. Целью настоящей работы является обобщение признака двумерного многообразия Веронезе на случай произвольной размерности.

Прежде всего напомним основные определения и факты из теории подмногообразий проективного пространства, необходимые для дальнейшего изложения.

(C) В.В. Коннов, 2000 
1.1. Отображение проективизации. Пусть $W$ - вещественное векторное пространство размерности $N+1, P^{N}=P(W)$ - порожденное им проективное пространство, $p: W \backslash 0 \rightarrow P(W)$ - каноническая проекция (отображение проективизации).

Используя любую аффинную карту на $P(W)$, легко проверить, что дифференциал $\left(p_{*}\right)_{a}: T_{a} W \rightarrow T_{p(a)} P(W)$ отображения проективизации $p$ в точке $a \in W \backslash 0$ обладает свойством: $\left(p_{*}\right)_{a}(b)=\left(p_{*}\right)_{a}(c) \Longleftrightarrow b \equiv c \bmod (a)$, где $b, c \in W \cong T_{a} W$. Следовательно, $\operatorname{ker}\left(p_{*}\right)_{a}=\langle a\rangle$. Здесь и далее через $\langle a, b, \ldots, c\rangle$ обозначается подпространство в $W$, являющееся линейной оболочкой векторов $a, b, \ldots, c$. Рассмотрим последовательность векторных пространств и их гомоморфизмов:

$$
0 \rightarrow\langle a\rangle \stackrel{i}{\rightarrow} T_{a} W \cong W \stackrel{\left(p_{*}\right)_{a}}{\longrightarrow} T_{p(a)} P(W) \rightarrow 0,
$$

где $i$ - гомоморфизм включения. Так как $\operatorname{Im} i=\operatorname{ker}\left(p_{*}\right)_{a}=\langle a\rangle$, то рассматриваемая последовательность является точной. Поэтому существует естественный изоморфизм между касательным векторным пространством $T_{p(a)} P(W)$ в точке $p(a)$ проективного пространства $P(W)$ и факторпространством $W /\langle a\rangle$ пространства $W$ по одномерному подпространству $\langle a\rangle$. Таким образом, $T_{p(a)} P(W) \cong W /\langle a\rangle$. В дальнейшем мы будем отождествлять эти векторные пространства.

1.2. Многообразие реперов проективного пространства. Обозначим через $\mathscr{F}(P(W))$ многообразие всех базисов $B=\left\{e_{0}, e_{1}, \ldots, e_{N}\right\}$ пространства $W$, нормированных условием $\operatorname{det}\left\{e_{0}, e_{1}, \ldots, e_{N}\right\}=1$ относительно некоторого фиксированного базиса $B_{0}$. Каждый базис $B=\left\{e_{u}\right\} \in \mathscr{F}(P(W))$ однозначно определяет проективный репер $\left\{E_{u}, E\right\}$ с вершинами $E_{u}=p\left(e_{u}\right)$ и единичной точкой $E=p\left(\sum_{u=0}^{N} e_{u}\right)$. Поэтому фиксация базиса $B_{0}$ позволяет отождествить многообразие $\mathscr{F}(P(W))$ с группой Ли $\mathrm{SL}(N+1)$, которая изоморфна связной компоненте единицы $\mathrm{PGL}_{0}(N)$ полной проективной группь $\mathrm{PGL}(N)$. Алгебра Ли $\mathfrak{p g r}(N)$ групп Ли $\mathrm{PGL}_{0}(N)$ и $\mathrm{PGL}(N)$ изоморфна алгебре $\mathfrak{s l}(N+1)$ бесследовых квадратных матриц порядка $N+1$.

Каждый вектор $e_{u}$ базиса $B \in \mathscr{F}(P(W))$ можно рассматривать как гладкую $W$-значную функцию $\left(e_{u}: B \longmapsto e_{u}\right)$ на группе Ли $\mathrm{PGL}_{0}(N)$. Поэтому $d e_{u}-$ это $W$-значная 1-форма на $\mathrm{PGL}_{0}(N)$. Раскладывая ее по базису $B$ пространства $W$, получим

$$
d e_{u}=\omega_{u}^{v} e_{v}
$$

Формы $\omega_{u}^{v}$ являются левоинвариантными формами групшы Ли $\operatorname{PGL}(N)$ и удовлетворяют структурным уравнениям Маурера-Картана:

$$
\begin{gathered}
d \omega_{u}^{v}=\omega_{u}^{w} \wedge \omega_{w}^{v}, \\
\omega_{0}^{0}+\omega_{1}^{1}+\cdots+\omega_{N}^{N}=0 .
\end{gathered}
$$

Уравнения (2) и (3) получаются после применения оператора внешнего дифференцирования к системе (1) и тождеству $\operatorname{det}\left\{e_{0}, e_{1}, \ldots, e_{N}\right\}=1$. Равенства (1), в которых 1-формы $\omega_{u}^{v}$ удовлетворяют уравнениям (2), (3), называются уравнениями инфинитезимальных перемещений репера проективного пространства, а сами уравнения (2), (3) - структурнылми уравнениями многообразия реперов проективного пространства [1]. Эти уравнения играют фундаментальную роль при изучении подмногообразий проективного пространства. 
ПРЕДЛОЖЕНИЕ 1 [3], [4]. Пусть $\omega_{u}^{v}-$ формы Маурера-Картана группы $\operatorname{PGL}(N)$ и $f_{1}, f_{2}: M \rightarrow \mathscr{F}(P(W))$ - два гладких отображсения связного многообразия $M$ в многообразие $\mathscr{F}(P(W))$. Равенство $f_{1}^{*} \omega_{u}^{v}=f_{2}^{*} \omega_{u}^{v}$ является необходимым и достаточнымм условием того, что $f_{1}(x)=g \cdot f_{2}(x)$ для фиксированного $g \in \mathrm{PGL}(N)$ и произвольного $x \in M$.

ПРЕДЛОЖЕНИЕ 2 [3], [4]. Пусть $\theta_{u}^{v}-$ дифференииальные 1-формы на связном и односвязном многообразии $M, \omega_{u}^{v}$ - формы Маурера-Картана группы $\operatorname{PGL}(N)$. Формы $\theta_{u}^{v}$ удовлетворяют уравнениям Маурера-Картана (2), (3) группь $\mathrm{PGL}(N)$ тогда и только тогда, когда существует гладкое отображение $f: M \rightarrow \mathscr{F}(P(W))$, для которого $\theta_{u}^{v}=f^{*} \omega_{u}^{v}$.

ЗАмЕчАниЕ 1 . В дальнейшем нам придется рассматривать проективные пространства $P^{N}=P(W)$ размерности $N=\left(\begin{array}{c}2 \\ n+2\end{array}\right)-1=n(n+3) / 2$, где $n \in \mathbb{N}$. В этом случае базисные векторы пространства $W$ удобно нумеровать симметричной парой индексов $(u, v)$, где $0 \leqslant u, v \leqslant n$. Для многообразия базисов $\mathscr{F}(P(W))$, состоящих из векторов $e_{u v}=e_{v u}$, уравнения (1)-(3) перепишутся в виде

$$
\begin{gathered}
d e_{u_{1} u_{2}}=\omega_{u_{1} u_{2}}^{v_{1} v_{2}} e_{v_{1} v_{2}}, \\
d \omega_{u_{1} u_{2}}^{v_{1} v_{2}}=\omega_{u_{1} u_{2}}^{w_{1} w_{2}} \wedge \omega_{w_{1} w_{2}}^{v_{1} v_{2}}, \\
\sum_{u_{1}, u_{2}=0}^{n} \omega_{u_{1} u_{2}}^{u_{1} u_{2}}=0,
\end{gathered}
$$

где $0 \leqslant u_{1}, u_{2}, v_{1}, v_{2}, w_{1}, w_{2} \leqslant n$.

1.3. Гладкие вложения в проективное пространство и их поднятия в векторное пространство. Пусть $f: M \rightarrow P(W)$-гладкое вложение $n$-мерного связного многообразия $M$ в проективное пространство $P(W)$; тогда $f(M)=V$ гладкое связное подмногообразие в $P(W)$.

Рассмотрим произвольное гладкое поднятие $F$ отображения $f$ в векторное пространство $W$ (напомним, что $F: M \rightarrow W$ - это гладкое отображение, удовлетворяюшее условию $f=p \circ F$ ). Поднятие $F$ существует для любой иммерсии $f$. Действительно, отождествим векторное пространство $W$ с евклидовым пространством $\mathbb{R}^{N+1}$ и будем рассматривать $N$-мерную сферу $S^{N}$ как совокупность единичных векторов в $W$. Тогда $S^{N}$ является универсальным накрывающим пространством для $P(W)$, а отображение проективизации $p$ есть двулистное накрытие. Ясно, что для иммерсии $f: M \rightarrow P(W)$ существует поднятие $F: M \rightarrow S^{N} \subset W$ на сферу $S^{N}$, которое является также и поднятием в $W$. Если теперь $\widetilde{F}: M \rightarrow W-$ другое поднятие для $f$, то для любой точки $x \in M$ векторы $F(x)$ и $\widetilde{F}(x)$ коллинеарны и отличны от нуля, так как лежат над одной точкой $f(x)$ пространства $P(W)$. Значит, $\widetilde{F}(x)=\lambda(x) \cdot F(x)$, где $\lambda$ - гладкая функция на $M$, всюду отличная от нуля (а поэтому сохраняюшая свой знак). Итак, допустимая замена поднятия определяется соотношением

$$
\widetilde{F}=\lambda \cdot F,
$$

где $\lambda$ - гладкая знакопостоянная функция на $M$. 
Для каждой карты $(U, \phi)$ на $M$ отображение $F_{U}=F \circ \phi^{-1}: \phi^{-1}(U) \rightarrow W$, являющееся локальньм представлением поднятия $F$, называется векторным уравнением подмногообразия $V$. Допустимая замена поднятия индуцирует перенормировку векторного уравнения. Если подмногообразие $V$ задано (локально) векторным уравнением, то проективный характер имеют лишь те его свойства, которые инвариантны относительно перенормировки этого векторного уравнения.

1.4. Последовательности производящих, касательных и нормальных подпространств. Фундаментальные формы подмногообразий. Пусть $f$ гладкое вложение $n$-мерного связного многообразия $M$ в проективное пространство $P(W), F$ - некоторое фиксированное поднятие отображения $f$ в векторное пространство $W$. С подмногообразием $V=f(M)=p \circ F(M) \subset P(W)$ можно связать ряд инвариантных объектов и отображений.

Пусть $A$ - некоторая точка на $V, f(x)=A$ и $F(x)=a$. Поскольку $f$ - диффеоморфизм на образ, то $\operatorname{Im}\left(f_{*}\right)_{x}=T_{A}(V)-n$-мерное подпространство векторного пространства $T_{A}(P(W)) \cong W /\langle a\rangle$. С другой стороны, так как $F-$ диффеоморфизм на образ, $\left(f_{*}\right)_{x}=\left(p_{*}\right)_{a} \circ\left(F_{*}\right)_{x}$ и $\operatorname{ker}\left(p_{*}\right)_{a}=\langle a\rangle$, то $\operatorname{Im}\left(F_{*}\right)_{x}-n$-мерное подпространство векторного пространства $T_{a}(W) \cong W$, трансверсальное подпространству $\langle a\rangle$. Поэтому $W_{A}^{1}(V)=\left\langle F(x), \operatorname{Im}\left(F_{*}\right)_{x}\right\rangle-(n+1)$-мерное подпространство векторного пространства $W$. Если $Q=F_{U}$ - векторное уравнение подмногообразия $V$, то

$$
W_{A}^{1}(V)=\left\langle F(x), \operatorname{Im}\left(F_{*}\right)_{x}\right\rangle=\left\langle Q(u), \frac{\partial Q(u)}{\partial x^{1}}, \frac{\partial Q(u)}{\partial x^{2}}, \ldots, \frac{\partial Q(u)}{\partial x^{n}}\right\rangle,
$$

где $(U, \phi)$ - карта на $M, u=\phi(x)$ и $f(x)=A$.

Докажем, что подпространство $W_{A}^{1}(V)$ не зависит от выбора поднятия, т.е. инвариантно определяется вложением $f$. Рассмотрим допустимую замену поднятия $\widetilde{F}=\lambda \cdot F$, и пусть $Q=F_{U}$ и $\widetilde{Q}=\widetilde{F}_{U}$ - локальные представления поднятий $F$ и $\widetilde{F}$ (т.е. $Q$ и $\widetilde{Q}$ - векторные уравнения подмногообразия $V$, заданные в области $\phi^{-1}(U)$ и совпадающие с точностью до перенормировки). Тогда

$$
\widetilde{Q}=\lambda \cdot Q, \quad \widetilde{Q}_{i}=\lambda_{i} \cdot Q+\lambda \cdot Q_{i},
$$

где $\widetilde{Q}_{i}=\frac{\partial \widetilde{Q}}{\partial x^{i}}, Q_{i}=\frac{\partial Q}{\partial x^{i}}$ и $\lambda_{i}=\frac{\partial \lambda}{\partial x^{i}}$. Так как матрица перехода

$$
\left(\begin{array}{ccccc}
\lambda & 0 & 0 & \ldots & 0 \\
\lambda_{1} & \lambda & 0 & \ldots & 0 \\
\lambda_{2} & 0 & \lambda & \ldots & 0 \\
\ldots & \ldots & \ldots & \ldots & . \\
\lambda_{n} & 0 & 0 & \ldots & \lambda
\end{array}\right)
$$

является невырожденной, то $\left\langle Q, Q_{1}, \ldots, Q_{n}\right\rangle=\left\langle\widetilde{Q}, \widetilde{Q}_{1}, \ldots, \widetilde{Q}_{n}\right\rangle$, и подпространство $W_{A}^{1}(V)$ не зависит от выбора поднятия.

Подпространство $W_{A}^{1}(V)$ назовем первыц производящим пространствомподмногообразия $V$ в точке $A$. Его образ при проективизации $p\left(W_{A}^{1}(V)\right)=P T_{A}^{1}(V)$ называется проективны.м касательны.м пространством подмногообразия $V$ в 
точке $A$, а факторпространство $W / W_{A}^{1}(V)=N_{A}^{1}(V)$ называется первыц м нормальным пространством подмногообразия $V$ в точке $A$. Заметим, что касательное векторное пространство $T_{A}^{1}(V)$ также может быть определено через первое производящее пространство: $T_{A}^{1}(V)=W_{A}^{1}(V) /\langle a\rangle$.

Поднятие $F: M \rightarrow W$ будем рассматривать как гладкую $W$-значную функцию на $M$. Пусть $\aleph(M)-C^{\infty}(M)$-модуль векторных полей на многообразии $M, x \in M$, $X_{i} \in \aleph(M)$. Так как $F$ - гладкая $W$-значная функция на $M$, а $X_{1}$ - дифференцирование алгебры гладких функций, то $X_{1}(F)=d F\left(X_{1}\right)$ - вновь гладкая $W$-значная функция на $M$. Аналогично, $X_{k}\left(\ldots\left(X_{1}(F)\right) \ldots\right)$ - гладкая $W$-значная функция на $M$, и ее значение $X_{k}\left(\ldots\left(X_{1}(F)\right) \ldots\right)(x)$ в точке $x \in M$ является элементом векторного пространства $W$. Для каждого $k \in \mathbb{N}$ и каждой точки $x \in M$ определим отображение

$$
d_{x}^{k} F: \underbrace{\aleph(M) \times \aleph(M) \times \cdots \times \aleph(M)}_{k} \rightarrow W
$$

следующим образом. Для векторных полей $X_{i} \in \aleph(M)$ положим

$$
d_{x}^{k} F\left(X_{1}, \ldots, X_{k}\right)=X_{k}\left(\ldots\left(X_{1}(F)\right) \ldots\right)(x) .
$$

Можно показать, что для любого $k \in \mathbb{N}$ и любой точки $A=f(x)=p(F(x))$ подпространство

$$
W_{A}^{k}(V)=\left\langle F(x), \operatorname{Im} d_{x}^{1} F, \ldots, \operatorname{Im} d_{x}^{k} F\right\rangle
$$

векторного пространства $W$ не зависит от выбора поднятия $F$ (инвариантно относительно перенормировки векторного уравнения) и определяется только вложением $f$. Подпространство $W_{A}^{k}(V)$ назовем производящим пространством порядка $k$ подмногообразия $V$ в точке $A$.

Если $Q=F_{U}$ - векторное уравнение подмногообразия $V$, то

$$
W_{A}^{k}(V)=\left\langle Q(u), \frac{\partial Q(u)}{\partial x_{i}}, \frac{\partial^{2} Q(u)}{\partial x_{j_{1}} \partial x_{j_{2}}}, \ldots, \frac{\partial^{k} Q(u)}{\partial x_{l_{1}} \ldots \partial x_{l_{k}}} \mid i, \ldots, l_{k}=1, \ldots, n\right\rangle,
$$

где $(U, \phi)$ - карта на $M, u=\phi(x)$ и $f(x)=A$.

Подпространство $P T_{A}^{k}(V)=p\left(W_{A}^{k}(V)\right)$ пространства $P(W)$ называется $n$ роективным касательным пространством порядка $k$ (или соприкасающимся пространством порядка $k-1)$ подмногообразия $V$ в точке $A$. Факторпространство $N_{A}^{k}(V)=W / W_{A}^{k}(V)$ называется нор.мальныцм пространством порядка $k$ подмногообразия $V$ в точке $A$.

Отображение

$$
\Psi_{A}^{k}: \operatorname{Sym} \underbrace{T_{x}(M) \times T_{x}(M) \times \cdots \times T_{x}(M)}_{k} \rightarrow N_{A}^{k-1}(V)
$$

определяемое формулой

$$
\boldsymbol{\Psi}_{A}^{k}\left(X_{1}, X_{2}, \ldots, X_{k}\right)=W_{A}^{k-1}(V)+d_{x}^{k} F\left(X_{1}, X_{2}, \ldots, X_{k}\right),
$$


называется $k$-ой фундаментальной формой подмногообразия $V$. В локальной системе координат это отображение имеет вид

$$
\Psi_{A}^{k}\left(X_{1}, X_{2}, \ldots, X_{k}\right)=W_{A}^{k-1}(V)+\frac{\partial^{k} Q(u)}{\partial x^{l_{1}} \ldots \partial x^{l_{k}}} X_{1}^{l_{1}} X_{2}^{l_{2}} \ldots X_{k}^{l_{k}}
$$

где $(U, \phi)$ - карта на $M, u=\phi(x)$ и $f(x)=A$. Легко проверить, что при замене карты на $M$ компоненты фундаментальной формы $\boldsymbol{\Psi}_{A}^{k}$ преобразуются по тензорному закону, а при замене поднятия - умножаются на число. Таким образом, форма $\Psi_{A}^{k}$ является относительным тензором на $M$ (а значит, и на V) со значениями в нормальном пространстве $N_{A}^{k-1}(V)$. Ранг этого тензора

$$
\begin{aligned}
\operatorname{rank} \Psi_{A}^{k} & \equiv \operatorname{dim}\left(\operatorname{Im} \Psi_{A}^{k}\right)=\operatorname{dim} W_{A}^{k}(V)-\operatorname{dim} W_{A}^{k-1}(V) \\
& =\operatorname{dim} P T_{A}^{k}(V)-\operatorname{dim} P T_{A}^{k-1}(V)=\operatorname{dim} N_{A}^{k-1}(V)-\operatorname{dim} N_{A}^{k}(V) \\
& =\operatorname{rank}\left\langle\frac{\partial^{k} Q(u)}{\partial x^{l_{1}} \ldots \partial x^{l_{k}}} \mid l_{1}, \ldots, l_{k}=1, \ldots, n\right\rangle
\end{aligned}
$$

является абсолютным инвариантом и полностью определяется вложением $f$.

Отметим, что в классической литературе по проективно-дифференциальной геометрии тензор $\Psi_{A}^{k}$ называется асимптотической формой порядка $k$. При $k=1$ форма $\Psi_{A}^{1}$ - это просто касательное отображение $\left(f_{*}\right)_{x}$, ранг которого равен $n$.

Можно показать, что условие $\Psi_{A}^{k} \equiv 0$ является необходимым и достаточным признаком того, что подмногообразие $V$ содержится в своем проективном касательном пространстве порядка $k-1$. В частности, тождество $\Psi_{A}^{2} \equiv 0$ является признаком того, что $V-n$-мерная проективная плоскость.

ЗАмечАниЕ 2. Проективное касательное пространство $P T_{A}^{k}(V)$ порядка $k$ (или, что то же самое, соприкасающееся пространство порядка $k-1$ ) подмногообразия $V \subset P^{N}$ можно определить несколько иначе. Пусть $A, A_{1}, A_{2}, \ldots, A_{k}$ - точки гладкой кривой $\gamma \subset P^{N}$. Предельное положение (если оно существует) проективной плоскости, натянутой на точки $A, A_{1}, A_{2}, \ldots, A_{k}$, при $A_{1}, A_{2}, \ldots, A_{k} \rightarrow A$ называется проективной касательной плоскостью порядка $k$ кривой $\gamma$ в точ$\kappa e A$. Объединение проективных касательных плоскостей порядка $k$ в точке $A$ ко всем кривым, лежашим на $V$ и проходящим через $A$, является подпространством в $P^{N}$, которое называется проективным касательным пространством порядка $k$ подмногообразия $V$ в точке $A$.

1.5. Расслоенные многообразия реперов над гладким подмногообразием. Пусть $P^{N}=P(W)$ - проективное пространство, а $\mathscr{F}(P(W))$ - многообразие реперов, определенное в п. 1.2.

Рассмотрим гладкую проекцию

$$
\pi_{0}=p \circ e_{0}: \mathscr{F}(P(W)) \rightarrow P(W),
$$

сопоставляющую каждому базису $B=\left\{e_{u}\right\} \in \mathscr{F}(P(W))$ точку $A_{0}=p\left(e_{0}\right)$, порожденную вектором $e_{0}$. Проекция (7) определяет главное расслоение с тоталь- 
ным пространством $\mathscr{F}(P(W))$, базой $P(W)$ и структурной группой $\mathrm{G}_{0}$, являющейся стационарной подгруппой точки $A_{0}$ :

$$
\begin{gathered}
\mathrm{G}_{0}=\left\{g \in \mathrm{PGL}_{0}(N) \mid \pi_{0}(B g)=\pi_{0}(B)=A_{0} \text { для любого } B \in \mathscr{F}(P(W))\right\} ; \\
\mathrm{G}_{0} \cong\left\{\left(\begin{array}{cc}
g_{0}^{0} & \overbrace{0 \ldots 0}^{N-1} \\
\left(g_{i}^{0}\right) & \left(g_{i}^{j}\right)
\end{array}\right) \in \mathrm{SL}(N+1)\right\} .
\end{gathered}
$$

Так как $\pi_{0}$ - гладкая сюръекция, то дифференциал $d_{B} \pi_{0}$ является эпиморфизмом на $T_{p\left(e_{0}\right)}(P(W)) \cong W /\left\langle e_{0}\right\rangle$. Поэтому $\operatorname{dim}\left(\operatorname{Im}\left(d_{B} \pi_{0}\right)\right)=N$. С другой стороны, для любого $X \in \aleph(\mathscr{F}(P(W)))$ имеем

$$
\begin{aligned}
d_{B} \pi_{0}(X) & =d_{e_{0}} p \circ d_{B} e_{0}(X) \\
& =\left(p_{*}\right) e_{0}\left(\omega_{0}^{0}(X) e_{0}+\omega_{0}^{1}(X) e_{1}+\omega_{0}^{2}(X) e_{2}+\cdots+\omega_{0}^{N}(X) e_{N}\right) \\
& =\left\langle e_{0}\right\rangle+\omega_{0}^{1}(X) \widetilde{e}_{1}+\omega_{0}^{2}(X) \widetilde{e}_{2}+\cdots+\omega_{0}^{N}(X) \widetilde{e}_{N},
\end{aligned}
$$

где $\tilde{e}_{i}=\left(p_{*}\right)_{e_{0}}\left(e_{i}\right), i=1,2, \ldots, N$. Следовательно, 1 -формы $\omega_{0}^{1}, \omega_{0}^{2}, \ldots, \omega_{0}^{N}$ линейно независимы и образуют базис пространства $T_{p\left(e_{0}\right)}^{*}(P(W))$. Эти формы являются горизонтальными для расслоения (7). Максимальными интегральными многообразиями вполне интегрируемой системы Пфаффа $\omega_{0}^{1}=\omega_{0}^{2}=\cdots=\omega_{0}^{N}=0$ являются слои расслоения (7), каждый из которых диффеоморфен $\mathrm{G}_{0}$ и состоит из всех реперов, лежащих над фиксированной точкой $A_{0}=\pi_{0}(B)$ пространства $P(W)$.

Пусть теперь $V$-гладкое связное $n$-мерное подмногообразие в проективном пространстве $P(W), f$ - отображение вложения. Обозначим через $\mathscr{F}^{0}(V)=\pi_{0}^{-1}(V)$ порцию над $V$ для расслоения (7). Тогда $\mathscr{F}^{0}(V)$ - гладкое связное подмногообразие в $\mathscr{F}(P(W))$, являющееся тотальным пространством для главного расслоения с базой $V$, проекцией $\pi_{0}$ и структурной группой $\mathrm{G}_{0}$. Многообразие $\mathscr{F}^{0}(V)$ называется многообразием реперов нулевого порядка над подмногообразием $V$.

Пусть $\hat{f}: \mathscr{F}^{0}(V) \rightarrow \mathscr{F}(P(W))$ - отображение вложения. При помоши оператора антиувлечения функции $e_{u}$ и формы $\omega_{u}^{v}$, определенные на $\mathscr{F}(P(W))$, переносятся на подмногообразие $\mathscr{F}^{0}(V)$. Обозначим: $\theta_{u}^{v}=\hat{f}^{*} \omega_{u}^{v}$. Для $W$-значных функций $\hat{f}^{*} e_{u}=e_{u} \circ \hat{f}$, заданных на $\mathscr{F}^{0}(V)$, сохраним прежние обозначения $e_{u}$. На многообразии реперов $\mathscr{F}^{0}(V)$ будут выполняться уравнения (1)-(3). Формы $\theta_{0}^{1}, \theta_{0}^{2}, \ldots, \theta_{0}^{N}$ будут являться горизонтальными на расслоении $\pi_{0}: \mathscr{F}^{0}(V) \rightarrow V$, причем $\operatorname{rank}\left\{\theta_{0}^{1}, \theta_{0}^{2}, \ldots, \theta_{0}^{N}\right\}=\operatorname{dim} V=n$.

Расслоенное многообразие реперов $\mathscr{F}^{0}(V)$ несет полную информацию о дифференциальной геометрии подмногообразия $V$. В частности, проекция $\pi_{0}=p \circ e_{0}$ позволяет находить фундаментальные формы $\Psi_{A}^{k}$ и инвариантные пространства, определенные в п. 1.4, без явного использования поднятия $F$. Действительно, если $F$ - произвольное фиксированное поднятие в пространство $W$ вложения $f$, то $W$-значные функции $F$ и $e_{0}$ связаны условием

$$
e_{0}(B)=\lambda \cdot F\left(f^{-1}\left(\pi_{0}(B)\right)\right),
$$

где $\lambda=\lambda(B)$ - знакопостоянная функция на $\mathscr{F}^{0}(V)$. Рассматривая отображения

$$
d_{B}^{k} e_{0}: \underbrace{\aleph\left(\mathscr{F}^{0}(V)\right) \times \aleph\left(\mathscr{F}^{0}(V)\right) \times \cdots \times \aleph\left(\mathscr{F}^{0}(V)\right)}_{k} \rightarrow W
$$


построенные по закону

$$
d_{B}^{k} e_{0}\left(X_{1}, \ldots, X_{k}\right)=X_{k}\left(\ldots\left(X_{1}\left(e_{0}\right)\right) \ldots\right)(B)
$$

для $B \in \mathscr{F}^{0}(V)$ и $X_{i} \in \aleph\left(\mathscr{F}^{0}(V)\right)$, найдем, что

$$
W_{A_{0}}^{k}(V)=\left\langle e_{0}(B), \operatorname{Im} d_{B}^{1} e_{0}, \ldots, \operatorname{Im} d_{B}^{k} e_{0}\right\rangle
$$

- производящее пространство порядка $k$ подмногообразия $V$ в точке $A_{0}$;

$$
P T_{A_{0}}^{k}(V)=p\left(W_{A_{0}}^{k}(V)\right)
$$

- проективное касательное пространство порядка $k$ подмногообразия $V$ в точке $A_{0}$;

$$
N_{A_{0}}^{k}(V)=W / W_{A_{0}}^{k}(V)
$$

- нормальное пространство порядка $k$ подмногообразия $V$ в точке $A_{0}$;

$$
\Psi_{A_{0}}^{k}\left(\widehat{X}_{1}, \widehat{X}_{2}, \ldots, \widehat{X}_{k}\right)=W_{A_{0}}^{k-1}(V)+d_{B}^{k}\left(X_{1}, X_{2}, \ldots, X_{k}\right)
$$

- фундаментальная форма порядка $k$. Здесь $\left(f^{-1} \circ \pi_{0}\right)(B)=x \in M$ и $\left(f^{-1} \circ \pi_{0}\right)_{*}\left(X_{i}\right)=\widehat{X}_{i} \in \aleph(M)$. По сушеству форма $\Psi_{A_{0}}^{k}$ задана на многообразии реперов $\mathscr{F}^{0}(V)$ :

$$
\Psi_{A_{0}}^{k}\left(X_{1}, X_{2}, \ldots, X_{k}\right)=W_{A_{0}}^{k-1}(V)+d_{B}^{k}\left(X_{1}, X_{2}, \ldots, X_{k}\right)
$$

Производяшие пространства, инвариантно связанные с вложением $f$, позволяют строить редукции расслоения $\mathscr{F}^{0}(V)$. Так, например, требование $\left\langle e_{0}, e_{1}, \ldots\right.$ $\left.\ldots, e_{n}\right\rangle=W_{A_{0}}^{1}(V)$ выделяет в $\mathscr{F}^{0}(V)$ подрасслоение $\mathscr{F}^{1}(V)$, называемое расслоением реперов первого порядка над подмногообразием $V$. Далее, если rank $\Psi_{A}^{2} \equiv$ $s$, то условие $\left\langle e_{0}, e_{1}, \ldots, e_{n+s}\right\rangle=W_{A_{0}}^{2}(V)$ определяет в $\mathscr{F}^{1}(V)$ подмногобразие $\mathscr{F}^{2}(V)$, назьваемое расслоением реперов второго порядка над подмногообрази-

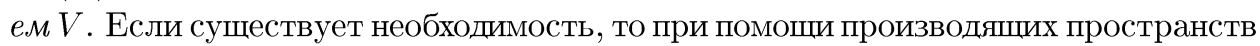
более высокого порядка можно продолжить процесс построения соответствующих подрасслоений:

$$
\mathscr{F}^{0}(V) \supseteq \mathscr{F}^{1}(V) \supseteq \mathscr{F}^{2}(V) \supseteq \mathscr{F}^{3}(V) \supseteq \cdots
$$

ЗАмЕчАниЕ 3. В ряде случаев, чтобы не загромождать обозначений, удобно не делать различия между производяшим пространством порядка $k$ и им порожденным проективным касательным пространством порядка $k$. В подобной ситуации будем использовать обозначение: $P T_{A}^{k}(V) \approx W_{A}^{k}(V)$. 


\section{§ 2. Признак квадратичных вложений Веронезе}

Пусть $P^{n}$ - вещественное проективное пространство размерности $n$ с однородными координатами $\left(X^{u}\right)=\left(X^{0}: X^{1}: \ldots: X^{n}\right)$. Пространство $P^{n}$ можно вложить в проективное пространство $P^{N}$ размерности $N=\left(\begin{array}{c}m \\ n+m\end{array}\right)-1$ по формулам

$$
\rho \cdot X^{u_{1} u_{2} \ldots u_{m}}=X^{u_{1}} X^{u_{2}} \ldots X^{u_{m}}, \quad 0 \leqslant u_{1} \leqslant u_{2} \leqslant \cdots \leqslant u_{m} \leqslant n .
$$

Здесь симметричные по всем индексам величины $X^{u_{1}} u_{2} \ldots u_{m}$ являются однородными координатами в $P^{N}$. Вложение $f_{n}^{m}: P^{n} \rightarrow P^{N}$, определяемое формулами (8), называется вложением Веронезе, а его образ $V_{n}^{m}=f_{n}^{m}\left(P^{n}\right) \subset P^{N}-$ многообразием Веронезе (или, подробнее, $n$-мерным многообразием Веронезе степени $m)$. Одномерные многообразия Веронезе называются кривыми Веронезе.

В дальнейшем мы будем рассматривать лишь квадратичные вложения Веронезе, т.е. вложения $f_{n}^{2}$ :

$$
\rho \cdot X^{u v}=X^{u} X^{v}, \quad 0 \leqslant u \leqslant v \leqslant n .
$$

В этом случае размерность объемлющего пространства $P^{N}$ равна

$$
N=\left(\begin{array}{c}
2 \\
n+2
\end{array}\right)-1=\frac{n(n+3)}{2} .
$$

Пусть $W-(n+1)$-мерное действительное векторное пространство, $S^{2}(W)=$ $\operatorname{Sym}(W \otimes W)$ - пространство симметрических двухвалентных контравариантных тензоров, $P^{N}=P\left(S^{2}(W)\right)$ - проективизация тензорного пространства $S^{2}(W)$, т.е. проективное пространство, порожденное векторным пространством $S^{2}(W)$. Обозначим через $\mathscr{T}=\left\{x \otimes x \in S^{2}(W) \mid x \in W \backslash 0\right\}$ подмножество простых абсолютно вырожденных ненулевых тензоров из $S^{2}(W)$. Тогда $V_{n}^{2}=p(\mathscr{T})$ - многообразие Веронезе, вложенное в проективное пространство $P\left(S^{2}(W)\right)$ размерности $N=\left(\begin{array}{c}2 \\ n+2\end{array}\right)-1=n(n+3) / 2$. Само квадратичное вложение Веронезе

$$
f_{n}^{2}: P(W) \rightarrow P(\operatorname{Sym}(W \otimes W))
$$

в бескоординатной форме определяется по закону

$$
f_{n}^{2}:\langle x\rangle \longmapsto\langle x \otimes x\rangle
$$

где $x \in W,\langle x\rangle \approx p(x) \in P(W)$ и $\langle x \otimes x\rangle \approx p(x \otimes x) \in V_{n}^{2} \subset P(\operatorname{Sym}(W \otimes W))$.

Учитывая отождествления

$$
\begin{aligned}
T_{\langle x\rangle}(P(W)) & \cong W /\langle x\rangle, \\
T_{\langle x \otimes x\rangle}(P(\operatorname{Sym}(W \otimes W))) & \cong \operatorname{Sym}(W \otimes W) /\langle x \otimes x\rangle,
\end{aligned}
$$

дифференциал

$$
d_{\langle x\rangle} f_{2}^{n}: T_{\langle x\rangle}(P(W)) \rightarrow T_{\langle x \otimes x\rangle}(P(\operatorname{Sym}(W \otimes W)))
$$


отображения $f_{n}^{2}$ можно рассматривать как мономорфизм

$$
d_{\langle x\rangle} f_{2}^{n}: W /\langle x\rangle \rightarrow \operatorname{Sym}(W \otimes W) /\langle x \otimes x\rangle
$$

Учитывая (10), можно убедиться, что это отображение задается по закону

$$
d_{\langle x\rangle} f_{2}^{n}:\langle x\rangle+y \longmapsto\langle x \otimes x\rangle+\frac{1}{2}(x \otimes y+y \otimes x)
$$

Следовательно, $\operatorname{Im}\left(d_{\langle x\rangle} f_{2}^{n}\right)=(x \otimes W+W \otimes x) /\langle x \otimes x\rangle$.

Итак,

$$
W_{\langle x \otimes x\rangle}^{1}\left(V_{n}^{2}\right)=x \otimes W+W \otimes x
$$

- первое производящее пространство многообразия Веронезе $V_{n}^{2}$ в точке $\langle x \otimes x\rangle$;

$$
T_{\langle x \otimes x\rangle}\left(V_{n}^{2}\right)=(x \otimes W+W \otimes x) /\langle x \otimes x\rangle
$$

- касательное векторное пространство многообразия Веронезе $V_{n}^{2}$ в точке $\langle x \otimes x\rangle$

$$
P T_{\langle x \otimes x\rangle}^{1}\left(V_{n}^{2}\right)=p(x \otimes W+W \otimes x) \approx\langle x \otimes W+W \otimes x\rangle
$$

- проективное касательное пространство многообразия Веронезе $V_{n}^{2}$ в точке $\langle x \otimes x\rangle$

$$
N_{\langle x \otimes x\rangle}^{1}\left(V_{n}^{2}\right)=\operatorname{Sym}(W \otimes W) /(x \otimes W+W \otimes x)
$$

- первое нормальное пространство многообразия Веронезе $V_{n}^{2}$ в точке $\langle x \otimes x\rangle$.

Отметим, что из (11) вытекает одно важное свойство квадратичных вложений Веронезе.

Свойство 1. Если $\langle x \otimes x\rangle u\langle y \otimes y\rangle-$ пара различных точек на многообразии Веронезе $V_{n}^{2}$, то проходящие через них проективные касательные плоскости $P T_{\langle x \otimes x\rangle}^{1}\left(V_{n}^{2}\right)$ и $P T_{\langle y \otimes y\rangle}^{1}\left(V_{n}^{2}\right)$ имеют единственную общую точку $\langle x \otimes y+y \otimes x\rangle$.

Многообразие Веронезе $V_{n}^{2}$ является простейшей орбитой относительно канонического действия групшы

$$
\mathrm{G}=\operatorname{diag}(\operatorname{PGL}(n) \times \operatorname{PGL}(n)) \cong \operatorname{PGL}(n)
$$

на пространстве $P\left(S^{2}(W)\right)$. Если $\left\{e_{0}, e_{1}, \ldots, e_{n}\right\}$ - базис векторного пространства $W$, то базис в $S^{2}(W)$ образуют тензоры $e_{u_{1} u_{2}}=e_{\left(u_{1}\right.} \otimes e_{\left.u_{2}\right)}$, где $u_{i}=$ $0,1, \ldots, n$. Таким образом, вложение Веронезе $f_{2}^{n}$ индуцирует гладкую инъекцию $\hat{s}_{n}^{2}: \mathscr{F}\left(P^{n}\right) \rightarrow \mathscr{F}\left(P^{N}\right)$ по закону $s_{n}^{2}:\left\{e_{u}\right\} \longmapsto\left\{e_{v w}\right\}$, где $e_{u v}=e_{(u} \otimes e_{v)}$. Пусть $\mathscr{F}\left(V_{n}^{2}\right)=\operatorname{Im}\left(s_{n}^{2}\right)$ - подмногообразие всех реперов из $\mathscr{F}\left(P^{N}\right)$, произведенных подобным образом. Каждый базис $B=\left\{e_{u_{1} u_{2}}\right\}$ из $\mathscr{F}\left(V_{n}^{2}\right)$ определяет проективный репер в $P^{N}$ с вершинами $A_{u_{1} u_{2}}=p\left(e_{u_{1} u_{2}}\right) \approx\left\langle e_{u_{1} u_{2}}\right\rangle$. Относительно этого репера многообразие Веронезе $V_{n}^{2}$ задается параметрическими уравнениями (9). В силу (11) проективная касательная плоскость $P T_{\langle x \otimes x\rangle}^{1}\left(V_{n}^{2}\right)$ в точке $\langle x \otimes x\rangle=\left\{X^{u v}\right\}=\left\{X^{u} X^{v}\right\}$ имеет уравнение

$$
\rho \cdot X^{u v}=X^{(u} Y^{v)}, \quad 0 \leqslant u \leqslant v \leqslant n,
$$


где $Y^{u}$ - параметры.

Проективный репер $\left\{A_{u v}\right\}$ из $\mathscr{F}\left(V_{n}^{2}\right)$ непосредственно связан с многообразием Веронезе $V_{n}^{2}$ :

$$
\begin{gathered}
A_{u u} \in V_{n}^{2}, \quad 0 \leqslant u \leqslant n ; \\
A_{u v}=P T_{A_{u u}}^{1}\left(V_{n}^{2}\right) \cap P T_{A_{v v}}^{1}\left(V_{n}^{2}\right), \quad u \neq v, \quad 0 \leqslant u, v \leqslant n .
\end{gathered}
$$

Для каждого значения $u=0,1, \ldots, n$ рассмотрим проекцию

$$
\pi_{u u}: \mathscr{F}\left(V_{n}^{2}\right) \rightarrow V_{n}^{2}
$$

сопоставляющую каждому базису $\left\{e_{u v}\right\}$ из $\mathscr{F}\left(V_{n}^{2}\right)$ точку $A_{u u}=p\left(e_{u u}\right)$ многообразия Веронезе $V_{n}^{2}$. Так как проекции $\pi_{u и}$ являются композициями гладких отображений $e_{u u}$ и $p$, то $\pi_{u u}$ - гладкие сюръекции. Каждое отображение $\pi_{u и}$ определяет главное расслоение с тотальным пространством $\mathscr{F}\left(V_{n}^{2}\right)$, базой $V_{n}^{2}$ и структурной группой $\mathrm{G}$, изоморфной стационарной подгруппе точки на $P^{n}$. Для подмногообразия $\mathscr{F}\left(V_{n}^{2}\right) \subset \mathscr{F}\left(P^{N}\right)$ обозначим через $\hat{f}_{n}^{2}$ соответствующее отображение вложения.

На многообразии $\mathscr{F}\left(P^{N}\right)$ выполняется система уравнений (4)-(6) (см. замечание 1 ). Обозначим через $\theta_{u_{1} u_{2}}^{v_{1} v_{2}}=\left(\hat{f}_{n}^{2}\right)^{*} \omega_{u_{1} u_{2}}^{v_{1} v_{2}}$ увлеченные формы и применим к уравнениям (4)-(6) оператор антиувлечения $\left(\hat{f}_{n}^{2}\right)^{*}$. В результате получим

$$
\begin{gathered}
d e_{u_{1} u_{2}}=\theta_{u_{1} u_{2}}^{v_{1} v_{2}} e_{v_{1} v_{2}}, \\
d \theta_{u_{1} u_{2}}^{v_{1} v_{2}}=\theta_{u_{1} u_{2}}^{w_{1} w_{2}} \wedge \theta_{w_{1} w_{2}}^{v_{1} v_{2}}, \\
\sum_{u_{1}, u_{2}=0}^{n} \theta_{u_{1} u_{2}}^{u_{1} u_{2}}=0,
\end{gathered}
$$

где $0 \leqslant u_{1}, u_{2}, v_{1}, v_{2}, w_{1}, w_{2} \leqslant n$. В этих формулах для увлеченных функций $e_{u v} \circ \hat{f}_{n}^{2}$ сохранено прежнее обозначение $e_{u v}$.

Дифференцируя тождество $e_{u_{1} u_{2}}=e_{\left(u_{1}\right.} \otimes e_{\left.u_{2}\right)}$, определяюшее $\mathscr{F}\left(V_{n}^{2}\right)$, найдем

$$
\theta_{u_{1} u_{2}}^{v_{1} v_{2}}=2 \cdot \omega_{\left(u_{1}\right.}^{\left(v_{1}\right.} \delta_{\left.u_{2}\right)}^{\left.v_{2}\right)}
$$

где 1 -формы $\omega_{u}^{v}$ удовлетворяют структурным уравнениям Маурера-Картана группы PGL $(n)$ (см. уравнения (2), (3) при $N=n)$.

Базис горизонтальных форм в расслоении $\pi_{u u}$ составляют 1-формы $\omega_{u}^{v}$, где $u$ фиксировано, $v \neq u$. Выберем одну из проекций, например $\pi_{00}$, и рассмотрим $S^{2}(W)$-значную функцию $e_{00}$. Вычислим ее дифференциал в точке $B=\left\{e_{u v}\right\}$ :

$$
d_{B}^{1} e_{00}\left(X_{1}\right)=2 \theta_{0}^{i}\left(X_{1}\right) e_{0 i} \quad \bmod \left\langle e_{00}\right\rangle
$$

Здесь и далее $i, j=1,2, \ldots, n$. Следовательно, $W_{A_{00}}^{1}\left(V_{2}^{n}\right)=\left\langle e_{00}, e_{01}, \ldots, e_{0 n}\right\rangle$. Далее, вычисляя второй дифференциал, получим

$$
d_{B}^{2}\left(X_{1}, X_{2}\right)=2 \theta_{0}^{i}\left(X_{1}\right) \theta_{0}^{j}\left(X_{2}\right) e_{i j} \bmod \left\langle e_{00}, e_{01}, \ldots, e_{0 n}\right\rangle .
$$


Таким образом,

$$
\begin{aligned}
\Psi_{A_{00}}^{2}\left(X_{1}, X_{2}\right) & =\left\langle e_{00}, e_{01}, \ldots, e_{0 n}\right\rangle+2 \theta_{0}^{i}\left(X_{1}\right) \theta_{0}^{j}\left(X_{2}\right) e_{i j}, \\
\operatorname{rank} \Psi_{A_{00}}^{2} & =\left(\begin{array}{c}
2 \\
n+1
\end{array}\right)=\frac{n(n+1)}{2}, \\
\operatorname{dim}\left(W_{A_{00}}^{2}\left(V_{n}^{2}\right)\right) & =\frac{n(n+1)}{2}+(n+1)=\frac{(n+1)(n+2)}{2} \\
& =N+1=\operatorname{dim}\left(S^{2}(W)\right), \\
W_{A_{00}}^{2}\left(V_{n}^{2}\right) & =S^{2}(W), \quad P T_{A_{00}}^{2}\left(V_{n}^{2}\right)=P^{N}=P\left(S^{2}(W)\right) .
\end{aligned}
$$

Из последних соотношений следует еше одно важное свойство квадратичных вложений Веронезе.

Свойство 2. В каждой точке многообразия Веронезе $V_{n}^{2}$ вторая фундаментальная форма имеет максимально возможный ранг $n(n+1) / 2$, т.е. соприкасающаяся плоскость совпадает с оббемлющим пространством.

Теперь докажем, что свойства 1 и 2 являются характеристическими для квадратичных вложений Веронезе.

ТЕорема. Пусть $V$ - гладкое связное $n$-мерное $(n \geqslant 2)$ многообразие, вложенное в вещественное проективное пространство $P^{N}(N=n(n+3) / 2) u$ удовлетворяющее условиям:

(A) касательные проективные пространства для любой пары точек подмногообразия $V$ пересекаются в точке;

(B) соприкасающееся проективное пространство в каждой точке подмногообразия $V$ совпадает с обгемлющим пространством $P^{N}$.

Тогда подмногообразие $V$ локально проективно эквивалентно многообразию Веронезе $V_{n}^{2}$. Если к тому же многообразие $V$ диффеоморфно $P^{n}$, то подмногообразия $V$ и $V_{n}^{2}$ совпадают с точностью до проективного преобразования пространства $P^{N}$.

ЗАмечАние 4. Условие (В) теоремы означает, что в каждой точке подмногообразия $V$ вторая фундаментальная форма имеет максимально возможный ранг $n(n+1) / 2$. Условие $(\mathrm{A})$ равносильно секантной вырожденности подмногообразия $V$ (см. [1], [4]). Таким образом, теорема утверждает, что $n$-мерными секантно вырожденными подмногообразиями в $P^{N}$ со второй фундаментальной формой максимального ранга могут быть только квадратичные многообразия Веронезе. Для двумерного случая $(n=2, N=5)$ эта теорема впервые была доказана Гиффитсом и Харрисом в работе [4]. Более простое доказательство для двумерного случая имеется в книге [1].

ДОКАЗАТЕЛЬСТВо ТЕОРЕМЫ. Условимся, что всюду в дальнейшем индексы будут принимать следующие значения:

$$
u, v, w, u_{1}, u_{2}, v_{1}, v_{2}, w_{1}, w_{2}=0,1, \ldots, n, \quad i, j, k, l, p, q, m, r=1,2, \ldots, n-1 .
$$

Пусть $V=f(M)$ - подмногообразие в $P^{N}=P(W)$, удовлетворяющее условиям теоремы. Здесь $f$ - отображение вложения, $W-(N+1)$-мерное векторное пространство, порождаюшее проективное пространство $P^{N}$. 
Если $P$ и $Q$ - любая пара различных точек на $V$, то по условию (А) теоремы сушествует единственная точка, являюшаяся пересечением проективных плоскостей $P T_{P}^{1}(V)$ и $P T_{Q}^{1}(V)$. Поэтому мы можем рассмотреть многообразие реперов $\mathscr{F}(V \odot V) \subset \mathscr{F}\left(P^{N}\right)$, определяемое условиями:

$$
\begin{gathered}
P=A_{00}=p\left(e_{00}\right) \in V, \quad Q=A_{n n}=p\left(e_{n n}\right) \in V \\
P T_{P}^{1}(V) \cap P T_{Q}^{1}(V)=A_{0 n}=p\left(e_{0 n}\right), \\
W_{P}^{1}(V)=\left\langle e_{00}, e_{01}, \ldots, e_{0 n}\right\rangle, \quad W_{Q}^{1}(V)=\left\langle e_{n n}, \ldots, e_{n 1}, e_{n 0}\right\rangle .
\end{gathered}
$$

Можно доказать, что многообразие $\mathscr{F}(V \odot V)$ является тотальным пространством для главного расслоения с базой $V \times V \backslash \operatorname{diag}(V \times V)$, проекцией $\pi_{00} \times \pi_{n n}:\left\{e_{u v}\right\} \longmapsto$ $A_{00} \times A_{n n}$ и структурной группой $\mathrm{G}_{0}$. Эта группа является подгруппой в группе квадратных матрищ порядка $N+1$. Если занумеровать строки и столбцы парой симметричных индексов, то группу $\mathrm{G}_{0}$ будут образовывать матрицы вида $\left(C_{u_{1} u_{2}}^{v_{1} v_{2}}\right)$, для которых

$$
\begin{gathered}
\operatorname{det}\left(C_{u_{1} u_{2}}^{v_{1} v_{2}}\right)=1, \\
C_{00}^{0 i}=C_{00}^{0 n}=C_{00}^{i j}=C_{00}^{i n}=C_{00}^{n n}=0, \\
C_{n n}^{n i}=C_{n n}^{n 0}=C_{n n}^{i j}=C_{n n}^{i 0}=C_{n n}^{00}=0, \\
C_{0 i}^{i j}=C_{0 i}^{i n}=C_{0 i}^{n n}=0, \quad C_{n i}^{i j}=C_{n i}^{i 0}=C_{n i}^{00}=0, \\
C_{0 n}^{00}=C_{0 n}^{0 i}=C_{0 n}^{i j}=C_{0 n}^{i n}=C_{0 n}^{00}=0 .
\end{gathered}
$$

Действие группы $\mathrm{G}_{0}$ на многообразии $\mathscr{F}(V \odot V)$ определяется по закону

$$
\left(\left\{e_{u_{1} u_{2}}\right\},\left(C_{u_{1} u_{2}}^{v_{1} v_{2}}\right)\right) \longmapsto\left\{e_{u_{1} u_{2}}^{\prime}\right\}, \quad \text { где } e_{u_{1} u_{2}}^{\prime}=C_{u_{1} u_{2}}^{v_{1} v_{2}} e_{v_{1} v_{2}} .
$$

Главное расслоение $\left(\mathscr{F}(V \odot V), V \times V \backslash \operatorname{diag}(V \times V), \pi_{00} \times \pi_{n n}, \mathrm{G}_{0}\right)$ обозначим через $\Pi_{0}$.

На многообразии $\mathscr{F}\left(P^{N}\right)$ вьполняется система уравнений (4)-(6) (см. замечание 1$)$. Пусть $\hat{f}: \mathscr{F}(V \odot V) \rightarrow\left(P^{N}\right)$ - отображение вложения. Сохранив для увлеченных функций $e_{u v} \circ \hat{f}$ прежнее обозначение $e_{u v}$ и обозначив $\theta_{u_{1} u_{2}}^{v_{1} v_{2}}=\hat{f}^{*} \omega_{u_{1} u_{2}}^{v_{1} v_{2}}$, применим к уравнениям (4)-(6) оператор антиувлечения $\hat{f}^{*}$. В результате получим уравнения (13)-(15).

Так как $W_{P}^{1}(V)=\operatorname{Im}\left(d_{B} e_{00}\right)$ и

$$
d_{B} e_{00}(X)=2 \theta_{00}^{00}(X) e_{00}+2 \theta_{00}^{01}(X) e_{01}+\cdots+2 \theta_{00}^{0 N}(X) e_{0 N}
$$

для любого $X \in \aleph(\mathscr{F}(V \odot V))$, то условие $W_{P}^{1}(V)=\left\langle e_{00}, e_{01}, \ldots, e_{0 n}\right\rangle$ равносильно системе дифференциальных уравнений

$$
\theta_{00}^{i j}=\theta_{00}^{i n}=\theta_{00}^{n n}=0 .
$$

Точно так же из условия $W_{Q}^{1}(V)=\left\langle e_{00}, e_{01}, \ldots, e_{0 n}\right\rangle$ находим

$$
\theta_{n n}^{i j}=\theta_{n n}^{i 0}=\theta_{n n}^{00}=0 .
$$


Базис горизонтальных форм расслоения $\Pi_{0}$ образуют 1-формы

$$
\theta_{0}^{1}=\theta_{00}^{01}, \theta_{0}^{2}=\theta_{00}^{02}, \ldots, \theta_{0}^{n}=\theta_{00}^{0 n}, \quad \theta_{n}^{0}=\theta_{n n}^{0 n}, \theta_{n}^{1}=\theta_{n n}^{1 n}, \ldots, \theta_{n}^{n-1}=\theta_{n n}^{n n-1} .
$$

При этом на расслоении $\pi_{00}: \mathscr{F}(V \odot V) \rightarrow V$ базис горизонтальных форм образуют 1 -формы $\theta_{0}^{1}, \theta_{0}^{2}, \ldots, \theta_{0}^{n}$, а на расслоении $\pi_{n n}: \mathscr{F}(V \odot V) \rightarrow V$ аналогичный базис состоит из форм $\theta_{n}^{0}, \theta_{n}^{1}, \ldots, \theta_{n}^{n-1}$.

Применяя оператор внешнего дифференцирования к системе уравнений (17), (18) и разрешая результат по лемме Картана, найдем

$$
\begin{array}{ll}
2 \theta_{0 k}^{i j}=A_{k l}^{i j} \theta_{0}^{l}+A_{k}^{i j} \theta_{0}^{n} ; \quad 2 \theta_{0 k}^{i n}=A_{k l}^{i} \theta_{0}^{l}+A_{k}^{i} \theta_{0}^{n} ; & 2 \theta_{0 k}^{n n}=A_{k l} \theta_{0}^{l}+A_{k} \theta_{0}^{n} \\
2 \theta_{0 n}^{i j}=A_{l}^{i j} \theta_{0}^{l}+A^{i j} \theta_{0}^{n} ; \quad 2 \theta_{0 n}^{i n}=A_{l}^{i} \theta_{0}^{l}+A^{i} \theta_{0}^{n} ; & 2 \theta_{0 n}^{n n}=A_{l} \theta_{0}^{l}+A \theta_{0}^{n} ; \\
2 \theta_{n k}^{i j}=\bar{A}_{k l}^{i j} \theta_{n}^{l}+\bar{A}_{k}^{i j} \theta_{n}^{0} ; 2 \theta_{n k}^{i 0}=\bar{A}_{k l}^{i} \theta_{n}^{l}+\bar{A}_{k}^{i} \theta_{n}^{0} ; & 2 \theta_{n k}^{00}=\bar{A}_{k l} \theta_{n}^{l}+\bar{A}_{k} \theta_{n}^{0} ; \\
2 \theta_{n 0}^{i j}=\bar{A}_{l}^{i j} \theta_{n}^{l}+\bar{A}^{i j} \theta_{n}^{0} ; \quad 2 \theta_{n 0}^{i 0}=\bar{A}_{l}^{i} \theta_{n}^{l}+\bar{A}^{i} \theta_{n}^{0} ; & 2 \theta_{n 0}^{00}=\bar{A}_{l} \theta_{n}^{l}+\bar{A} \theta_{n}^{0} .
\end{array}
$$

Здесь величины $A_{k l}^{i j}, \bar{A}_{k l}^{i j}, A_{k}^{i j}, \bar{A}_{k}^{i j}, A_{k l}^{j}, \bar{A}_{k l}^{j}, A_{i j}, \bar{A}_{i j}, A_{k}^{i}, \bar{A}_{k}^{i}, A_{k}^{j}, \bar{A}_{k}^{j}, A_{k}, \bar{A}_{k}, A^{j}$, $\bar{A}^{j}, A, \bar{A}$ являются гладкими функциями на $\mathscr{F}(V \odot V)$. Величины $A_{k l}^{i j}, \bar{A}_{k l}^{i j}$ симметричны по нижним и верхним индексам, $A_{k}^{i j}, \bar{A}_{k}^{i j}, A^{i j}, \bar{A}^{i j}$ - по верхним индексам, $A_{k l}^{j}, \bar{A}_{k l}^{j}, A_{i j}, \bar{A}_{i j}-$ по нижним индексам. Сравнивая первые серии уравнений в $(20)$ и (22) и учитывая линейную независимость 1 -форм $\theta_{0}^{l}, \theta_{0}^{n}, \theta_{n}^{l}$ и $\theta_{n}^{0}$, найдем

$$
\begin{aligned}
A^{i j}=\bar{A}^{i j}=A_{k}^{i j} & =\bar{A}_{k}^{i j}=0, \\
\theta_{0 n}^{i j} & =0 .
\end{aligned}
$$

Так как для любых векторов $X_{1}$ и $X_{2}$, касательных к многообразию $\mathscr{F}(V \odot V)$ в точке $B=\left\{e_{u v}\right\}$, имеют место равенства

$$
\begin{aligned}
d_{B} e_{00}\left(X_{1}\right)= & 2\left(\theta_{0}^{i}\left(X_{1}\right) e_{0 i}+\theta_{0}^{n}\left(X_{1}\right) e_{0 n}\right) \quad \bmod \left\langle e_{00}\right\rangle, \\
d_{B}^{2} e_{00}\left(X_{1}, X_{2}\right)= & \theta_{0}^{i}\left(X_{1}\right) \theta_{0}^{j}\left(X_{2}\right)\left(A_{i j}^{k l} e_{k l}+2 A_{i j}^{k} e_{n k}+A_{i j} e_{n n}\right) \\
& +\left(\theta_{0}^{i}\left(X_{1}\right) \theta_{0}^{n}\left(X_{2}\right)+\theta_{0}^{i}\left(X_{2}\right) \theta_{0}^{n}\left(X_{1}\right)\right)\left(2 A_{i}^{k} e_{k n}+A_{i} e_{n n}\right) \\
& +\theta_{0}^{n}\left(X_{1}\right) \theta_{0}^{n}\left(X_{2}\right) A e_{n n} \quad \bmod \left\langle e_{00}, e_{01}, \ldots, e_{0 n}\right\rangle
\end{aligned}
$$

то $\operatorname{rank}\left(\Psi_{P}^{2}\right)=\operatorname{dim}\left\langle\varepsilon_{i j}, \varepsilon_{n i}, \varepsilon_{n n}\right\rangle$, где $\varepsilon_{i j}=A_{i j}^{k l} e_{k l}+2 A_{i j}^{k} e_{n k}+A_{i j} e_{n n}, \varepsilon_{n i}=$ $2 A_{i}^{k} e_{k n}+A_{i} e_{n n}, \varepsilon_{n n}=A e_{n n}$.

По условию (В) теоремы $P T_{P}^{2}(V)=P^{N}$, т.е. $\operatorname{rank}\left(\mathbf{\Psi}_{P}^{2}\right)=n(n+1) / 2$. Это возможно лишь в том случае, если векторы $\varepsilon_{i j}, \varepsilon_{n k}, \varepsilon_{n n}$ линейно независимы. Поэтому $A \neq 0,\left(A_{i}^{j}\right)$ - невырожденная $(n-1)$-матрица, а $\left(A_{i j}^{k l}\right)$ - невырожденная $(n(n-1) / 2)$-матрица. Аналогично, вычисляя вторую фундаментальную форму $\Psi_{Q}^{2}$ в точке $Q=p\left(e_{n n}\right)$, убеждаемся в том, что $\bar{A} \neq 0$ и $\left(\bar{A}_{i}^{j}\right),\left(\bar{A}_{i j}^{k l}\right)-$ невырожденные матрицы. В силу вьшесказанного сушествуют матрицы $\left(B_{i}^{j}\right),\left(B_{i j}^{k l}\right),\left(\bar{B}_{i}^{j}\right)$ и $\left(\bar{B}_{i j}^{k l}\right)$, определенные условиями

$$
A_{i}^{j} B_{j}^{k}=\bar{A}_{i}^{j} \bar{B}_{j}^{k}=\delta_{i}^{k}, \quad A_{i j}^{k l} B_{k l}^{p q}=\bar{A}_{i j}^{k l} \bar{B}_{k l}^{p q}=\delta_{i j}^{p q}=\delta_{(i}^{(p} \delta_{j)}^{q)}=\frac{1}{2}\left(\delta_{i}^{p} \delta_{j}^{q}+\delta_{i}^{q} \delta_{j}^{p}\right) .
$$


Применим оператор внешнего дифференцирования к равенствам (24). В результате получим

$$
\left(\bar{A}_{l}^{k} A_{k s}^{i j}-A_{s}^{k} \bar{A}_{k l}^{i j}\right) \theta_{n}^{l} \wedge \theta_{0}^{s}+\bar{A}^{k} A_{k l}^{i j} \theta_{n}^{0} \wedge \theta_{0}^{l}+A^{k} \bar{A}_{k l}^{i j} \theta_{0}^{n} \wedge \theta_{n}^{l}=0 .
$$

Учитывая линейную независимость 1-форм $\theta_{0}^{l}, \theta_{0}^{n}, \theta_{n}^{l}$ и $\theta_{n}^{0}$, найдем

$$
\bar{A}_{l}^{k} A_{k s}^{i j}=A_{s}^{k} \bar{A}_{k l}^{i j}, \quad \bar{A}^{k} A_{k l}^{i j}=0, \quad A^{k} \bar{A}_{k l}^{i j}=0 .
$$

В силу невырожденности матриц $\left(A_{i j}^{k l}\right)$ и $\left(\bar{A}_{i j}^{k l}\right)$ из вторых и третьих серий равенств системы (26) следуют соотношения

$$
\bar{A}^{k}=A^{k}=0 .
$$

Преобразуем первую систему равенств $(26)$, свернув их с $B_{p}^{s}$ :

$$
\bar{A}_{p l}^{i j}=B_{p}^{s} \bar{A}_{l}^{k} A_{k s}^{i j} .
$$

Так как величины $\bar{A}_{p l}^{i j}$ симметричны по нижним индексам, то $B_{[p}^{s} \bar{A}_{l]}^{k} A_{k s}^{i j}=0$. Свернув эти равенства с $B_{i j}^{m r}$, получим $B_{[p}^{s} \bar{A}_{l]}^{k} \delta_{k}^{(m} \delta_{s}^{r)}=0$. Следовательно, $B_{[p}^{(m} \bar{A}_{l]}^{r)}=0$, т.e.

$$
B_{p}^{m} \bar{A}_{l}^{r}-B_{l}^{m} \bar{A}_{p}^{r}+B_{p}^{r} \bar{A}_{l}^{m}-B_{l}^{r} \bar{A}_{p}^{m}=0 .
$$

Обозначив $\bar{A}_{i}^{j} A_{j}^{i}=\alpha$ и свернув последние равенства с $A_{r}^{l}$, найдем

$$
\bar{A}_{p}^{m}=\frac{\alpha}{n-1} B_{p}^{m} .
$$

Итак, имеет место следующая система тождеств:

$$
\bar{A}_{i}^{j} A_{j}^{i}=\alpha, \quad \bar{A}_{i}^{k} A_{k}^{j}=\frac{\alpha}{n-1} \delta_{i}^{j}, \quad \bar{A}_{k l}^{i j}=\frac{n-1}{\alpha} \bar{A}_{k}^{s} \bar{A}_{l}^{p} A_{s p}^{i j} .
$$

Теперь с учетом (27) из (20), (22) находим, что $\theta_{0 n}^{i n}=\frac{1}{2} A_{l}^{i} \theta_{0}^{l}$ и $\theta_{n 0}^{i 0}=\frac{1}{2} \bar{A}_{l}^{i} \theta_{n}^{l}$. После дифференцирования получим

$$
\begin{aligned}
& \left(d A_{j}^{i}+A_{j}^{i}\left(\theta_{00}^{00}-2 \theta_{0 n}^{0 n}\right)-2 A_{k}^{i} \theta_{0 j}^{0 k}+2 A_{j}^{k} \theta_{n k}^{n i}-\bar{A}_{p}^{k} A_{k j}^{i} \theta_{n}^{p}-A_{j} \theta_{n}^{i}\right) \wedge \theta_{0}^{j} \\
& \quad+\left(A \delta_{j}^{i}-2 \bar{A}_{j}^{k} A_{k}^{i}\right) \theta_{n}^{j} \wedge \theta_{0}^{n}=0 \\
& \left(d \bar{A}_{j}^{i}+\bar{A}_{j}^{i}\left(\theta_{n n}^{n n}-2 \theta_{n 0}^{n 0}\right)-2 \bar{A}_{k}^{i} \theta_{n j}^{n k}+2 \bar{A}_{j}^{k} \theta_{0 k}^{0 i}-A_{p}^{k} \bar{A}_{k j}^{i} \theta_{0}^{p}-\bar{A}_{j} \theta_{0}^{j}\right) \wedge \theta_{n}^{j} \\
& \quad+\left(\bar{A} \delta_{j}^{i}-2 A_{j}^{k} \bar{A}_{k}^{i}\right) \theta_{0}^{j} \wedge \theta_{n}^{0}=0 .
\end{aligned}
$$

Следовательно,

$$
\begin{gathered}
d A_{j}^{i}+A_{j}^{i}\left(\theta_{00}^{00}-2 \theta_{0 n}^{0 n}\right)-2 A_{k}^{i} \theta_{0 j}^{0 k}+2 A_{j}^{k} \theta_{n k}^{n i}=C_{j k}^{i} \theta_{0}^{k}+C_{j}^{i} \theta_{0}^{n}, \\
d \bar{A}_{j}^{i}+\bar{A}_{j}^{i}\left(\theta_{n n}^{n n}-2 \theta_{n 0}^{n 0}\right)-2 \bar{A}_{k}^{i} \theta_{n j}^{n k}+2 \bar{A}_{j}^{k} \theta_{0 k}^{0 i}=\bar{C}_{j k}^{i} \theta_{n}^{k}+\bar{C}_{j}^{i} \theta_{n}^{0}, \\
\bar{C}_{[j k]}^{i}=C_{[j k]}^{i}=0, \quad A \delta_{j}^{i}-2 \bar{A}_{j}^{k} A_{k}^{i}=\bar{A} \delta_{j}^{i}-2 A_{j}^{k} \bar{A}_{k}^{i}=0, \quad A=\bar{A} .
\end{gathered}
$$


В силу этого равенства (28) запишутся в виде

$$
\bar{A}_{i}^{j} A_{j}^{i}=\frac{n-1}{2} A, \quad \bar{A}_{i}^{k} A_{k}^{j}=\frac{1}{2} A \delta_{i}^{j}, \quad \bar{A}_{k l}^{i j}=\frac{2}{A} \bar{A}_{k}^{s} \bar{A}_{l}^{p} A_{s p}^{i j} .
$$

Дифференцирование равенств $\theta_{n 0}^{n n}=\frac{1}{2}\left(A_{i} \theta_{0}^{i}+A \theta_{0}^{n}\right)$ и $\theta_{0 n}^{00}=\frac{1}{2}\left(\bar{A}_{i} \theta_{n}^{i}+A \theta_{n}^{0}\right)$ приводит к соотношениям

$$
\begin{aligned}
d A= & A\left(4 \theta_{0 n}^{0 n}-\theta_{00}^{00}-\theta_{n n}^{n n}\right)+\bar{A}_{i} A_{j}^{i} \theta_{0}^{j}+A_{i} \bar{A}_{j}^{i} \theta_{n}^{j}, \\
d A_{l}- & A_{l}\left(2 \theta_{n 0}^{n 0}-\theta_{00}^{00}-\theta_{n n}^{n n}\right)-2 A_{j} \theta_{0 l}^{0 j} \\
& -2 A \theta_{0 l}^{0 n}+2 A_{l}^{i} \theta_{n i}^{n n}-\bar{A}_{j}^{i} A_{i l} \theta_{n}^{j}=C_{l j} \theta_{0}^{j}, \\
d \bar{A}_{l}- & \bar{A}_{l}\left(2 \theta_{0 n}^{0 n}-\theta_{00}^{00}-\theta_{n n}^{n n}\right)-2 \bar{A}_{j} \theta_{n l}^{n j} \\
& -2 \bar{A} \theta_{n l}^{n 0}+2 \bar{A}_{l}^{i} \theta_{0 i}^{00}-A_{j}^{i} \bar{A}_{i l} \theta_{0}^{j}=\bar{C}_{l j} \theta_{n}^{j},
\end{aligned}
$$

где $C_{[l j]}=\bar{C}_{[l j]}=0$. Учитьвая (29), (30) и (32), продифференцируем вторую серию равенств системы (31). В результате придем к системе соотношений:

$C_{i}^{j}=\bar{C}_{i}^{j}=0, \quad A C_{i j}^{k}=\bar{A}_{(i}^{(k} \bar{A}_{j)}^{l)} A_{l}+\bar{A}_{i}^{l} \bar{A}_{j}^{p} \bar{A}_{q}^{k} A_{l p}^{q}, \quad A \bar{C}_{i j}^{k}=A_{(i}^{(k} A_{j)}^{l)} \bar{A}_{l}+A_{i}^{l} A_{j}^{p} A_{q}^{k} \bar{A}_{l p}^{q}$. Следовательно, уравнения (29), (30) примут вид

$$
\begin{gathered}
d A_{j}^{i}+A_{j}^{i}\left(\theta_{00}^{00}-2 \theta_{0 n}^{0 n}\right)-2 A_{k}^{i} \theta_{0 j}^{0 k}+2 A_{j}^{k} \theta_{n k}^{n i} \\
=\frac{1}{A}\left(\bar{A}_{(j}^{(i} \bar{A}_{k)}^{l)} A_{l}+\bar{A}_{j}^{l} \bar{A}_{k}^{p} \bar{A}_{q}^{i} A_{l p}^{q}\right) \theta_{0}^{k}, \\
d \bar{A}_{j}^{i}+\bar{A}_{j}^{i}\left(\theta_{n n}^{n n}-2 \theta_{n 0}^{n 0}\right)-2 \bar{A}_{k}^{i} \theta_{n j}^{n k}+2 \bar{A}_{j}^{k} \theta_{0 k}^{0 i} \\
=\frac{1}{A}\left(A_{(j}^{(i} A_{k)}^{l)} \bar{A}_{l}+A_{j}^{l} A_{k}^{p} A_{q}^{i} \bar{A}_{l p}^{q}\right) \theta_{n}^{k} .
\end{gathered}
$$

Последовательно применяя оператор внешнего дифференцирования и лемму Картана к равенствам

$$
\begin{gathered}
\theta_{0 k}^{i j}=\frac{1}{2} A_{k l}^{i j} \theta_{0}^{l}, \quad \theta_{n k}^{i j}=\frac{1}{2} \bar{A}_{k l}^{i j} \theta_{n}^{l}, \\
\theta_{0 k}^{i n}=\frac{1}{2} A_{k l}^{i} \theta_{0}^{l}+\frac{1}{2} A_{k}^{i} \theta_{0}^{n}, \quad \theta_{n k}^{i 0}=\frac{1}{2} \bar{A}_{k l}^{i} \theta_{n}^{l}+\frac{1}{2} \bar{A}_{k}^{i} \theta_{n}^{0}, \\
\theta_{0 k}^{n n}=\frac{1}{2} A_{k l} \theta_{0}^{l}+\frac{1}{2} A_{k} \theta_{0}^{n}, \quad \theta_{n k}^{00}=\frac{1}{2} \bar{A}_{k l} \theta_{n}^{l}+\frac{1}{2} \bar{A}_{k} \theta_{n}^{0},
\end{gathered}
$$

придем к следуюшей системе дифференциальных уравнений:

$$
\begin{aligned}
& d A_{k l}^{i j}+A_{k l}^{i j} \theta_{00}^{00}+A_{k l}^{p q} \theta_{p q}^{i j}-2 A_{l p}^{i j} \theta_{0 k}^{0 p}-2 A_{k p}^{i j} \theta_{0 l}^{0 p}=C_{k l s}^{i j} \theta_{0}^{s}-A_{k l}^{p} \bar{A}_{p s}^{i j} \theta_{n}^{s}, \\
& d \bar{A}_{k l}^{i j}+\bar{A}_{k l}^{i j} \theta_{n n}^{n n}+\bar{A}_{k l}^{p q} \theta_{p q}^{i j}-2 \bar{A}_{l p}^{i j} \theta_{n k}^{n p}-2 \bar{A}_{k p}^{i j} \theta_{n l}^{n p}=\bar{C}_{k l s}^{i j} \theta_{n}^{s}-\bar{A}_{k l}^{p} A_{p s}^{i j} \theta_{0}^{s}, \\
& d A_{j k}^{i}-2 A_{l j}^{i} \theta_{0 k}^{0 l}-2 A_{k l}^{i} \theta_{0 j}^{0 l}+2 A_{k j}^{l} \theta_{l n}^{i n}+A_{k j}^{i} \theta_{00}^{00} \\
& +A_{k j}^{l p} \theta_{l p}^{i n}-2 A_{j}^{i} \theta_{0 k}^{0 n}-2 A_{k}^{i} \theta_{0 j}^{0 n}+2 A_{k j} \theta_{n}^{i}=C_{j k l}^{i} \theta_{0}^{l}, \\
& d \bar{A}_{j k}^{i}-2 \bar{A}_{l j}^{i} \theta_{n k}^{n l}-2 \bar{A}_{k l}^{i} \theta_{n j}^{n l}+2 \bar{A}_{k j}^{l} \theta_{l 0}^{i 0}+\bar{A}_{k j}^{i} \theta_{n n}^{n n} \\
& +\bar{A}_{k j}^{l p} \theta_{l p}^{i 0}-2 \bar{A}_{j}^{i} \theta_{n k}^{n 0}-2 \bar{A}_{k}^{i} \theta_{n j}^{n 0}+2 \bar{A}_{k j} \theta_{0}^{i}=\bar{C}_{j k l}^{i} \theta_{n}^{l}, \\
& d A_{i j}+A_{i j}\left(\theta_{00}^{00}+\theta_{n n}^{n n}\right)-2 A_{i l} \theta_{0 j}^{0 l}-2 A_{j l} \theta_{0 i}^{0 l}+A_{i j}^{k l} \theta_{k l}^{n n}-2 A_{j} \theta_{0 i}^{0 n}-2 A_{i} \theta_{0 j}^{0 n} \\
& =C_{i j k} \theta_{0}^{k}+C_{i j} \theta_{0}^{n} \\
& d \bar{A}_{i j}+\bar{A}_{i j}\left(\theta_{n n}^{n n}+\theta_{00}^{00}\right)-2 \bar{A}_{i l} \theta_{n j}^{n l}-2 \bar{A}_{j l} \theta_{n i}^{n l}+\bar{A}_{i j}^{k l} \theta_{k l}^{00}-2 A_{j} \theta_{n i}^{0 n}-2 \bar{A}_{i} \theta_{n j}^{n 0} \\
& =\bar{C}_{i j k} \theta_{n}^{k}+\bar{C}_{i j} \theta_{n}^{0} \text {. }
\end{aligned}
$$


Новые величины $C_{k l p}^{i j}, \bar{C}_{k l p}^{i j}, C_{j k l}^{i}, \bar{C}_{j k l}^{i}, C_{j k l}$ и $\bar{C}_{j k l}$, входящие в систему (37)-(42) и появившиеся после применения леммы Картана, удовлетворяют очевидным условиям симметрии.

Тождества

$$
A=\bar{A}, \quad \bar{A}_{i}^{k} A_{k}^{j}=\frac{1}{2} A \delta_{i}^{j}, \quad \bar{A}_{k l}^{i j}=\frac{2}{A} \bar{A}_{k}^{s} \bar{A}_{l}^{p} A_{s p}^{i j}
$$

будут играть фундаментальную роль в дальнейшем доказательстве. В частности, будет использован тот факт, что

$$
\frac{2}{A} \bar{A}_{i}^{k} \bar{A}_{j}^{l} A_{k l}^{p q} e_{p q}=\bar{A}_{i j}^{p q} e_{p q}
$$

для любого базиса $\left\{e_{00}, e_{n n}, e_{0 i}, e_{n i}, e_{0 n}, e_{i j}\right\}$, лежащего над парой точек $P \times Q$. Эти тождества позволяют одновременно привести к диагональному виду пару фундаментальных форм $\boldsymbol{\Psi}_{P}^{2}(V)$ и $\boldsymbol{\Psi}_{Q}^{2}(V)$ в точках $P$ и $Q$.

Рассмотрим допустимую замену базиса, лежащего над парой точек $P \times Q$ :

$$
\left\{\begin{array}{l}
e_{00}^{\prime}=\rho \cdot \frac{A}{2} e_{00} ; \quad e_{n n}^{\prime}=\rho \cdot \frac{A^{2}}{4} e_{n n} ; \quad e_{0 n}^{\prime}=\rho \cdot \frac{A}{2} e_{0 n} ; \\
e_{0 i}^{\prime}=\rho \cdot\left(\bar{A}_{i}^{j} e_{0 j}+\frac{1}{2} \bar{A}_{i} e_{00}\right) ; \quad e_{n i}^{\prime}=\rho \cdot \bar{A}_{i}^{j}\left(A_{j}^{k} e_{k n}+\frac{1}{2} \bar{A}_{j} e_{n n}\right) \\
e_{i j}^{\prime}=\rho \cdot \frac{1}{2}\left(\bar{A}_{i j}^{p q} e_{p q}+2 \bar{A}_{i j}^{p} e_{0 p}+\bar{A}_{i j} e_{00}+\bar{A}_{i}^{k} \bar{A}_{j}^{l}\left(2 A_{k l}^{p} e_{p n}+A_{k l} e_{n n}\right)\right) .
\end{array}\right.
$$

Здесь множитель $\rho$ однозначно определяется из условия равенства единище определителя матрицы перехода. Данное преобразование принадлежит фундаментальной групе $\mathrm{G}_{0}$ расслоения $\Pi_{0}$. Действительно, оно невырождено, сохраняет точки $P=p\left(e_{00}\right)$ и $Q=p\left(e_{n n}\right)$, сохраняет проективные касательные плоскости $P T_{P}^{1}(V)$ и $P T_{Q}^{1}(V)$ вместе с их точкой пересечения $A_{0 n}=p\left(e_{0 n}\right)$.

Вычислим компоненты фундаментальных форм $\boldsymbol{\Psi}_{P}^{2}(V)$ и $\boldsymbol{\Psi}_{Q}^{2}(V)$ в новом базисе $\left\{e_{u v}^{\prime}\right\}$. Пусть $d e_{u_{1} u_{2}}^{\prime}=\bar{\theta}_{u_{1} u_{2}}^{v_{1} v_{2}} e_{v_{1} v_{2}}^{\prime}$. Из равенств

$$
\begin{aligned}
d e_{00}^{\prime} & =2\left(A_{i}^{j} \theta_{0}^{j} e_{0 j}^{\prime}+\theta_{0}^{n} e_{0 n}^{\prime}\right) \bmod \left\langle e_{00}^{\prime}\right\rangle, \\
d e_{n n}^{\prime} & =2\left(\frac{A}{2} \theta_{n}^{i} e_{n i}^{\prime}+\frac{A}{2} \theta_{n}^{0} e_{n 0}^{\prime}\right) \bmod \left\langle e_{n n}^{\prime}\right\rangle
\end{aligned}
$$

находим: $\bar{\theta}_{0}^{i}=A_{j}^{i} \theta_{0}^{j}, \bar{\theta}_{0}^{n}=\theta_{0}^{n}, \bar{\theta}_{n}^{i}=\frac{A}{2} \theta_{n}^{i}, \bar{\theta}_{n}^{0}=\frac{A}{2} \theta_{n}^{0}$. Далее, непосредственная проверка показывает, что

$$
\begin{aligned}
& d e_{0 i}^{\prime}=\bar{\theta}_{0}^{j} e_{i j}^{\prime}+\bar{\theta}_{0}^{n} e_{i n}^{\prime} \bmod \left\langle e_{00}^{\prime}, e_{0 i}^{\prime}, e_{0 n}^{\prime}\right\rangle, \\
& d e_{i n}^{\prime}=\bar{\theta}_{n}^{j} e_{i j}^{\prime}+\bar{\theta}_{n}^{0} e_{i 0}^{\prime} \bmod \left\langle e_{n n}^{\prime}, e_{n i}^{\prime}, e_{n 0}^{\prime}\right\rangle, \\
& d e_{0 n}^{\prime}=\bar{\theta}_{n}^{0} e_{00}^{\prime}+\bar{\theta}_{n}^{i} e_{0 i}^{\prime}+\bar{\theta}_{0}^{i} e_{n i}^{\prime}+\bar{\theta}_{0}^{n} e_{n n}^{\prime} \bmod \left\langle e_{0 n}^{\prime}\right\rangle .
\end{aligned}
$$

Следовательно,

$$
\begin{gathered}
\bar{\theta}_{00}^{i j}=\bar{\theta}_{00}^{i n}=\bar{\theta}_{00}^{n n}=\bar{\theta}_{n n}^{i j}=\bar{\theta}_{n n}^{i 0}=\bar{\theta}_{n n}^{00}=\bar{\theta}_{0 k}^{n n}=\bar{\theta}_{n k}^{00}=\bar{\theta}_{n 0}^{i j}=0, \\
\bar{\theta}_{0 k}^{i j}=\delta_{(k}^{(i} \delta_{l)}^{j)} \bar{\theta}_{0}^{l}, \quad \bar{\theta}_{0 k}^{i n}=\frac{1}{2} \delta_{k}^{i} \bar{\theta}_{0}^{n}, \quad \bar{\theta}_{n k}^{i 0}=\frac{1}{2} \delta_{k}^{i} \bar{\theta}_{n}^{0}, \quad \bar{\theta}_{n k}^{i j}=\delta_{(k}^{(i} \delta_{l)}^{j)} \bar{\theta}_{n}^{l}, \\
\bar{\theta}_{0 n}^{i n}=\frac{1}{2} \bar{\theta}_{0}^{i}, \quad \bar{\theta}_{0 n}^{n n}=\bar{\theta}_{0}^{n}, \quad \bar{\theta}_{n 0}^{00}=\bar{\theta}_{n}^{0}, \quad \bar{\theta}_{n 0}^{i 0}=\frac{1}{2} \bar{\theta}_{n}^{i} .
\end{gathered}
$$


Сравнивая систему (19)-(22) с системой (44)-(46), приходим к выводу, что после замены базиса по формулам (43) компоненты фундаментальных форм $\boldsymbol{\Psi}_{P}^{2}(V)$ и $\boldsymbol{\Psi}_{Q}^{2}(V)$ приводятся к виду

$$
\begin{gathered}
A_{k}^{i j}=\bar{A}_{k}^{i j}=A_{i j}^{k}=\bar{A}_{i j}^{k}=A^{i j}=\bar{A}^{i j}=A_{i j}=\bar{A}_{i j}=A^{i}=\bar{A}^{i}=A_{i}=\bar{A}_{i}=0 \\
A_{k l}^{i j}=\bar{A}_{k l}^{i j}=2 \delta_{(k}^{(i} \delta_{l)}^{j)}, \quad A_{j}^{i}=\bar{A}_{j}^{i}=\delta_{i}^{j}, \quad A=\bar{A}=2 .
\end{gathered}
$$

Обозначим через $\mathrm{G}_{1}$ подгруппу в $\mathrm{G}_{0}$, сохраняющую систему равенств $(47)$, и рассмотрим редукцию структурной группы $\mathrm{G}_{0}$ расслоения $\Pi_{0}$ к подгруппе $\mathrm{G}_{1}$. Coответствуюшее подрасслоение обозначим через $\Pi_{1}$, а соответствующее тотальное пространство, являюшееся интегральным многообразием системы Пфаффа (44)(46), обозначим через $\mathscr{F}_{1}(V \odot V)$. Сохраним символ $\hat{f}$ для отображения вложения $\mathscr{F}_{1}(V \odot V)$ в $\mathscr{F}\left(P^{N}\right)$, увлеченные формы $\hat{f}^{*} \theta_{u}^{v}$ и увлеченные функции $e_{u v} \circ \hat{f}$ будем снова обозначать через $\theta_{u}^{v}$ и $e_{u v}$ соответственно. В частности, так как базис $\left\{e_{u v}^{\prime}\right\}$, построенный по формулам (43), входит в $\mathscr{F}_{1}(V \odot V)$, то векторы $e_{u v}^{\prime}$ будем обозначать снова через $e_{u v}$, а для 1-форм $\bar{\theta}_{u}^{v}$, входящих в систему (44)-(46), сохраним обозначение $\theta_{u}^{v}$.

Первые дифференциальные следствия системы (44)-(46) получим прямой подстановкой выражений (47) в систему дифференциальных уравнений (32)-(42):

$$
\begin{gathered}
4 \theta_{0 n}^{0 n}-\theta_{00}^{00}-\theta_{n n}^{n n}=0, \\
2 \theta_{n l}^{n n}-4 \theta_{0 l}^{0 n}=C_{l j} \theta_{0}^{j}, \\
2 \theta_{0 l}^{00}-4 \theta_{n l}^{n 0}=\bar{C}_{l j} \theta_{n}^{j}, \\
\delta_{j}^{i}\left(\theta_{00}^{00}-2 \theta_{0 n}^{0 n}\right)-2 \theta_{0 j}^{0 i}+2 \theta_{n j}^{n i}=C_{j k}^{i} \theta_{0}^{k}, \\
\delta_{j}^{i}\left(\theta_{n n}^{n n}-2 \theta_{n 0}^{n 0}\right)-2 \theta_{n j}^{n i}+2 \theta_{0 j}^{0 i}=\bar{C}_{j k}^{i} \theta_{n}^{k}, \\
2 \delta_{k l}^{i j} \theta_{00}^{00}+2 \theta_{k l}^{i j}-4 \delta_{l p}^{i j} \theta_{0 k}^{0 p}-4 \delta_{k p}^{i j} \theta_{0 l}^{0 p}=C_{k l s}^{i j} \theta_{0}^{s}, \\
2 \delta_{k l}^{i j} \theta_{n n}^{n n}+2 \theta_{k l}^{i j}-4 \delta_{l p}^{i j} \theta_{n k}^{n p}-4 \delta_{k p}^{i j} \theta_{n l}^{n p}=\bar{C}_{k l s}^{i j} \theta_{n}^{s}, \\
2 \theta_{k j}^{i n}-2 \delta_{j}^{i} \theta_{0 k}^{0 n}-2 \delta_{k}^{i} \theta_{0 j}^{0 n}=C_{j k l}^{i} \theta_{0}^{l}, \\
2 \theta_{k j}^{i 0}-2 \delta_{j}^{i} \theta_{n k}^{n 0}-2 \delta_{k}^{i} \theta_{n j}^{n 0}=\bar{C}_{j k l}^{i} \theta_{n}^{l}, \\
2 \theta_{i j}^{n n}=C_{i j k} \theta_{0}^{k}+C_{i j} \theta_{0}^{n}, \\
2 \theta_{i j}^{00}=\bar{C}_{i j k} \theta_{n}^{k}+\bar{C}_{i j} \theta_{n}^{0} .
\end{gathered}
$$

Складывая уравнения (51), (52) и учитывая (48), найдем, что $C_{i j}^{k}=\bar{C}_{i j}^{k}=0$. Вычитая из равенства (51) равенство (52), получим

$$
4\left(\theta_{n i}^{n j}-\theta_{0 i}^{0 j}\right)=\delta_{i}^{j}\left(\theta_{n n}^{n n}-\theta_{00}^{00}\right) .
$$

Находя разность уравнений (53) и (54) и учитывая равенства (59), получим, что $C_{k l s}^{i j}=\bar{C}_{k l s}^{i j}=0$. Поэтому уравнения $(53),(54)$ запишутся в виде

$$
\theta_{k l}^{i j}=2\left(\delta_{l p}^{i j} \theta_{0 k}^{0 p}+\delta_{p k}^{i j} \theta_{0 l}^{0 p}\right)-\delta_{k l}^{i j} \theta_{00}^{00}=2\left(\delta_{l p}^{i j} \theta_{n k}^{n p}+\delta_{p k}^{i j} \theta_{n l}^{n p}\right)-\delta_{k l}^{i j} \theta_{n n}^{n n} .
$$


Дифференцируя равенство (48), получим, что $C_{i j}=\bar{C}_{i j}$. Следовательно,

$$
2 \theta_{n l}^{n n}-4 \theta_{0 l}^{0 n}=C_{l j} \theta_{0}^{j}, \quad 2 \theta_{0 l}^{00}-4 \theta_{n l}^{n 0}=C_{l j} \theta_{n}^{j} .
$$

Дифференцирование уравнений (61) и использование леммы Картана и уравнений (57), (58) приводят к соотношениям

$$
\begin{aligned}
d C_{i j}+\frac{1}{2} C_{i j}\left(3 \theta_{00}^{00}+\theta_{n n}^{n n}\right)-2 C_{i k} \theta_{0 j}^{0 k}-2 C_{k j} \theta_{0 i}^{0 k}-4 \theta_{i j}^{0 n}=C_{i j k} \theta_{n}^{l}+\lambda_{i p l} \theta_{0}^{l}, \\
d C_{i j}+\frac{1}{2} C_{i j}\left(3 \theta_{n n}^{n n}+\theta_{00}^{00}\right)-2 C_{i k} \theta_{n j}^{n k}-2 C_{k j} \theta_{n i}^{n k}-4 \theta_{i j}^{n 0}=\bar{C}_{i j k} \theta_{0}^{l}+\bar{\lambda}_{i p l} \theta_{n}^{l},
\end{aligned}
$$

где $\lambda_{i p l}$ и $\bar{\lambda}_{i p l}$-некоторые функции, симметричные по всем индексам. Вычитая эти уравнения одно из другого и используя соотношения (59), найдем, что $\lambda_{i p l}=\bar{C}_{i j k}$ и $\bar{\lambda}_{i p l}=C_{i j k}$. Следовательно,

$$
\begin{aligned}
d C_{i j}+ & C_{i j}\left(\theta_{n n}^{n n}+\theta_{00}^{00}\right)-C_{i k}\left(\theta_{n j}^{n k}+\theta_{0 j}^{0 k}\right)-C_{k j}\left(\theta_{0 i}^{0 k}+\theta_{n i}^{n k}\right)-4 \theta_{i j}^{n 0} \\
& =\bar{C}_{i j k} \theta_{0}^{l}+C_{i p l} \theta_{n}^{l} .
\end{aligned}
$$

Дифференцируя теперь равенства (59), найдем

$$
C_{j k l}^{i}+\bar{C}_{j k l}^{i}=\delta_{j}^{i} C_{k l}+\delta_{k}^{i} C_{l j}+\delta_{l}^{i} C_{j k} .
$$

Учитывая тождество (63) и дифференцируя систему равенств (60), придем к соотношениям

$$
\begin{aligned}
C_{k l p}^{i} \delta_{q}^{j}+ & C_{k l p}^{j} \delta_{q}^{i}+C_{l p q}^{i} \delta_{k}^{j}+C_{l p q}^{j} \delta_{k}^{i}+C_{p q k}^{i} \delta_{l}^{j}+C_{p q k}^{j} \delta_{l}^{i}+C_{q k l}^{i} \delta_{p}^{j}+C_{q k l}^{j} \delta_{p}^{i} \\
= & \delta_{k}^{i} \delta_{l}^{j} C_{p q}+\delta_{k}^{j} \delta_{l}^{i} C_{p q}+\delta_{l}^{i} \delta_{p}^{j} C_{q k}+\delta_{l}^{j} \delta_{p}^{i} C_{q k}+\delta_{p}^{i} \delta_{q}^{j} C_{k l}+\delta_{p}^{j} \delta_{q}^{i} C_{k l} \\
& +\delta_{q}^{i} \delta_{k}^{j} C_{l p}+\delta_{q}^{j} \delta_{k}^{i} C_{l p}+\delta_{k}^{i} \delta_{p}^{j} C_{l q}+\delta_{k}^{j} \delta_{p}^{i} C_{l q}+\delta_{l}^{i} \delta_{q}^{j} C_{k p}+\delta_{l}^{j} \delta_{q}^{i} C_{k p} \\
\bar{C}_{k l p}^{i} \delta_{q}^{j}+ & \bar{C}_{k l p}^{j} \delta_{q}^{i}+\bar{C}_{l p q}^{i} \delta_{k}^{j}+\bar{C}_{l p q}^{j} \delta_{k}^{i}+\bar{C}_{p q k}^{i} \delta_{l}^{j}+\bar{C}_{p q k}^{j} \delta_{l}^{i}+\bar{C}_{q k l}^{i} \delta_{p}^{j}+\bar{C}_{q k l}^{j} \delta_{p}^{i} \\
= & \delta_{k}^{i} \delta_{l}^{j} C_{p q}+\delta_{k}^{j} \delta_{l}^{i} C_{p q}+\delta_{l}^{i} \delta_{p}^{j} C_{q k}+\delta_{l}^{j} \delta_{p}^{i} C_{q k}+\delta_{p}^{i} \delta_{q}^{j} C_{k l}+\delta_{p}^{j} \delta_{q}^{i} C_{k l} \\
& +\delta_{q}^{i} \delta_{k}^{j} C_{l p}+\delta_{q}^{j} \delta_{k}^{i} C_{l p}+\delta_{k}^{i} \delta_{p}^{j} C_{l q}+\delta_{k}^{j} \delta_{p}^{i} C_{l q}+\delta_{l}^{i} \delta_{q}^{j} C_{k p}+\delta_{l}^{j} \delta_{q}^{i} C_{k p}
\end{aligned}
$$

При помощи операции свертки легко показать, что общее решение системы (63)(65) имеет вид

$$
C_{j k l}^{i}=\bar{C}_{j k l}^{i}=\frac{1}{2}\left(\delta_{j}^{i} C_{k l}+\delta_{k}^{i} C_{l j}+\delta_{l}^{i} C_{j k}\right) .
$$

Теперь уравнения (55), (56) запишутся в виде

$$
\begin{aligned}
& \theta_{k j}^{i n}-\delta_{j}^{i} \theta_{0 k}^{0 n}-\delta_{k}^{i} \theta_{0 j}^{0 n}=\frac{1}{4}\left(\delta_{j}^{i} C_{k l}+\delta_{k}^{i} C_{l j}+\delta_{l}^{i} C_{j k}\right) \theta_{0}^{l}, \\
& \theta_{k j}^{i 0}-\delta_{j}^{i} \theta_{n k}^{n 0}-\delta_{k}^{i} \theta_{n j}^{n 0}=\frac{1}{4}\left(\delta_{j}^{i} C_{k l}+\delta_{k}^{i} C_{l j}+\delta_{l}^{i} C_{j k}\right) \theta_{n}^{l} .
\end{aligned}
$$

Дифференцируя их и используя уравнения (62), получим

$\delta_{j}^{i} C_{k l p}+\delta_{k}^{i} C_{l p j}+\delta_{l}^{i} C_{p j k}+\delta_{p}^{i} C_{j k l}=0, \quad \delta_{j}^{i} \bar{C}_{k l p}+\delta_{k}^{i} \bar{C}_{l p j}+\delta_{l}^{i} \bar{C}_{p j k}+\delta_{p}^{i} \bar{C}_{j k l}=0$. 
Свертка этих соотношений приводит к равенствам

$$
C_{k l p}=\bar{C}_{k l p}=0 \text {. }
$$

Поэтому уравнения (57), (58) примут вид

$$
2 \theta_{i j}^{n n}=C_{i j} \theta_{0}^{n}, \quad 2 \theta_{i j}^{00}=C_{i j} \theta_{n}^{0} .
$$

Итак, доказано, что на многообразии реперов $\mathscr{F}_{1}(V \odot V)$ выполняется система дифференциальных уравнений, состояшая из $(48),(59)-(61),(66)-(68)$ и уравнений

$$
d C_{i j}+C_{i j}\left(\theta_{n n}^{n n}+\theta_{00}^{00}\right)-C_{i k}\left(\theta_{n j}^{n k}+\theta_{0 j}^{0 k}\right)-C_{k j}\left(\theta_{0 i}^{0 k}+\theta_{n i}^{n k}\right)-4 \theta_{i j}^{n 0}=0 .
$$

Проверка критерия Фробениуса показывает, что эта система является вполне интегрируемой.

Рассмотрим преобразование

$$
\left\{\begin{array}{l}
e_{00}^{\prime}=e_{00} ; \quad e_{n n}^{\prime}=e_{n n} ; \quad e_{0 n}^{\prime}=e_{0 n} ; \quad e_{0 i}^{\prime}=e_{0 i} ; \quad e_{n i}^{\prime}=e_{n i} \\
e_{i j}^{\prime}=e_{i j}-\frac{1}{2} C_{i j} e_{0 n} .
\end{array}\right.
$$

Сразу отметим, что определитель матрищы перехода для этого преобразования равен единице. Пусть $d e_{u_{1} u_{2}}^{\prime}=\bar{\theta}_{u_{1} u_{2}}^{v_{1} v_{2}} e_{v_{1} v_{2}}^{\prime}$. Очевидная проверка показывает, что для 1-форм $\bar{\theta}_{u_{1} u_{2}}^{v_{1} v_{2}}$ будет выполняться система дифференциальных уравнений (44)-(46). Следовательно, преобразование (70) принадлежит структурной группе $\mathrm{G}_{1}$ расслоения $\Pi_{1}$. В новом базисе система уравнений $(48),(59)-(61),(66)-(69)$ примет вид

$$
\left\{\begin{array}{l}
4 \bar{\theta}_{0 n}^{0 n}-\bar{\theta}_{00}^{00}-\bar{\theta}_{n n}^{n n}=0 \\
4\left(\bar{\theta}_{n i}^{n j}-\bar{\theta}_{0 i}^{0 j}\right)=\delta_{i}^{j}\left(\bar{\theta}_{n n}^{n n}-\bar{\theta}_{00}^{00}\right) \\
\bar{\theta}_{k l}^{i j}=2\left(\delta_{l p}^{i j} \bar{\theta}_{0 k}^{0 p}+\delta_{p k}^{i j} \bar{\theta}_{0 l}^{0 p}\right)-\delta_{k l}^{i j} \bar{\theta}_{00}^{00}=2\left(\delta_{l p}^{i j} \bar{\theta}_{n k}^{n p}+\delta_{p k}^{i j} \bar{\theta}_{n l}^{n p}\right)-\delta_{k l}^{i j} \bar{\theta}_{n n}^{n n} \\
\bar{\theta}_{n l}^{n n}-2 \bar{\theta}_{0 l}^{0 n}=\bar{\theta}_{0 l}^{00}-2 \bar{\theta}_{n l}^{n 0}=0 \\
\bar{\theta}_{k j}^{i n}-\delta_{j}^{i} \bar{\theta}_{0 k}^{0 n}-\delta_{k}^{i} \bar{\theta}_{0 j}^{0 n}=\bar{\theta}_{k j}^{i 0}-\delta_{j}^{i} \bar{\theta}_{n k}^{n 0}-\delta_{k}^{i} \bar{\theta}_{n j}^{n 0}=0 \\
\bar{\theta}_{i j}^{n n}=\bar{\theta}_{i j}^{00}=\bar{\theta}_{i j}^{n 0}=0 .
\end{array}\right.
$$

Эти уравнения показывают, в частности, что после преобразования (70) объект $C_{i j}$ стал равен нулю.

Обозначим через $\mathscr{F}_{2}(V \odot V)$ подмногообразие реперов из $\mathscr{F}_{1}(V \odot V)$, относительно которых $C_{i j}=0$, и пусть $\mathrm{G}_{2}$ - подгруппа в $\mathrm{G}_{1}$, которая сохраняет эти условия. Ясно, что четверка

$$
\Pi_{2}=\left(\mathscr{F}_{2}(V \odot V), V \times V \backslash \operatorname{diag}(V \times V), \pi_{00} \times \pi_{n n}, \mathrm{G}_{2}\right)
$$

- подрасслоение в $\Pi_{1}$. Тотальное пространство $\mathscr{F}_{2}(V \odot V)$ этого расслоения является максимальным интегральным подмногообразием вполне интегрируемой системы Пфаффа (44)-(46), (71), заданной на $\mathscr{F}\left(P^{N}\right)$. 
Введем обозначения:

$$
\left\{\begin{array}{l}
\omega_{0}^{0}=\frac{1}{2} \bar{\theta}_{00}^{00}, \quad \omega_{0}^{i}=\bar{\theta}_{0}^{i}, \quad \omega_{0}^{n}=\bar{\theta}_{0}^{n} \\
\omega_{i}^{0}=\bar{\theta}_{0 i}^{00}, \quad \omega_{i}^{j}=2 \bar{\theta}_{0 i}^{0 j}-\frac{1}{2} \delta_{i}^{j} \bar{\theta}_{00}^{00}, \quad \omega_{i}^{n}=\bar{\theta}_{n i}^{n n} \\
\omega_{n}^{0}=\bar{\theta}_{n}^{0}, \quad \omega_{n}^{i}=\bar{\theta}_{n}^{i}, \quad \omega_{n}^{n}=\frac{1}{2} \bar{\theta}_{n n}^{n n}
\end{array}\right.
$$

где $1 \leqslant i, j \leqslant n-1$. Тогда легко проверяется, что система уравнений (44)-(46), (71) перепишется в виде

$$
\bar{\theta}_{u_{1} u_{2}}^{v_{1} v_{2}}=2 \cdot \omega_{\left(u_{1}\right.}^{\left(v_{1}\right.} \delta_{\left.u_{2}\right)}^{\left.v_{2}\right)}, \quad 0 \leqslant u_{1}, u_{2}, v_{1}, v_{2} \leqslant n
$$

В силу (14) имеем

$$
d \bar{\theta}_{u_{1} u_{2}}^{v_{1} v_{2}}=\bar{\theta}_{u_{1} u_{2}}^{w_{1} w_{2}} \wedge \bar{\theta}_{w_{1} w_{2}}^{v_{1} v_{2}}, \quad 0 \leqslant u_{1}, u_{2}, v_{1}, v_{2}, w_{1}, w_{2} \leqslant n
$$

Следовательно,

$$
2 \cdot d \omega_{\left(u_{1}\right.}^{\left(v_{1}\right.} \delta_{\left.u_{2}\right)}^{\left.v_{2}\right)}=4 \cdot \omega_{\left(u_{1}\right.}^{\left(w_{1}\right.} \delta_{\left.u_{2}\right)}^{\left.w_{2}\right)} \wedge \omega_{\left(w_{1}\right.}^{\left(v_{1}\right.} \delta_{\left.w_{2}\right)}^{\left.v_{2}\right)}
$$

Преобразуем правую часть этого тождества:

$$
\begin{aligned}
& 4 \cdot \omega_{\left(u_{1}\right.}^{\left(w_{1}\right.} \delta_{\left.u_{2}\right)}^{\left.w_{2}\right)} \wedge \omega_{\left(w_{1}\right.}^{\left(v_{1}\right.} \delta_{\left.w_{2}\right)}^{\left.v_{2}\right)}=\left(\omega_{\left(u_{1}\right.}^{w_{1}} \delta_{\left.u_{2}\right)}^{w_{2}}+\omega_{\left(u_{1}\right.}^{w_{2}} \delta_{\left.u_{2}\right)}^{w_{1}}\right) \wedge\left(\omega_{w_{1}}^{\left(v_{1}\right.} \delta_{w_{2}}^{\left.v_{2}\right)}+\omega_{w_{2}}^{\left(v_{1}\right.} \delta_{w_{1}}^{\left.v_{2}\right)}\right) \\
& =\delta_{\left(u_{1}\right.}^{\left(v_{1}\right.} \omega_{\left.u_{2}\right)}^{\left|w_{2}\right|} \wedge \omega_{w_{2}}^{\left.v_{2}\right)}+2 \omega_{\left(u_{2}\right.}^{\left(v_{1}\right.} \wedge \omega_{\left.u_{1}\right)}^{\left.v_{2}\right)}+\delta_{\left(u_{1}\right.}^{\left(v_{1}\right.} \omega_{\left.u_{2}\right)}^{\left|w_{1}\right|} \wedge \omega_{w_{1}}^{\left.v_{2}\right)}=2 \delta_{\left(u_{1}\right.}^{\left(v_{1}\right.} \omega_{\left.u_{2}\right)}^{|w|} \wedge \omega_{w}^{\left.v_{2}\right)}
\end{aligned}
$$

Следовательно, $\delta_{\left(u_{1}\right.}^{\left(v_{1}\right.} d \omega_{\left.u_{2}\right)}^{\left.v_{2}\right)}=\delta_{\left(u_{1}\right.}^{\left(v_{1}\right.} \omega_{\left.u_{2}\right)}^{|w|} \wedge \omega_{w}^{\left.v_{2}\right)}$, т.е. $\delta_{\left(u_{1}\right.}^{\left(v_{1}\right.}\left(d \omega_{\left.u_{2}\right)}^{\left.v_{2}\right)}-\omega_{\left.u_{2}\right)}^{|w|} \wedge \omega_{w}^{\left.v_{2}\right)}\right)=0$.

Пусть $\Omega_{u}^{v}=d \omega_{u}^{v}-\omega_{u}^{w} \wedge \omega_{w}^{v}$. Тогда $\delta_{\left(u_{1}\right.}^{\left(v_{1}\right.} \Omega_{\left.u_{2}\right)}^{\left.v_{2}\right)}=0$, или подробнее:

$$
\delta_{u_{1}}^{v_{1}} \Omega_{u_{2}}^{v_{2}}+\delta_{u_{1}}^{v_{2}} \Omega_{u_{2}}^{v_{1}}+\delta_{u_{2}}^{v_{1}} \Omega_{u_{1}}^{v_{2}}+\delta_{u_{2}}^{v_{2}} \Omega_{u_{1}}^{v_{1}}=0
$$

Сворачивая найденные соотношения по индексам $u_{2}$ и $v_{2}$, получим

$$
(n+1) \Omega_{u_{1}}^{v_{1}}+\delta_{u_{1}}^{v_{1}} \Omega_{w}^{w}=0
$$

Повторно применяя операцию свертки, убеждаемся в том, что $\Omega_{w}^{w}=0$, а следовательно, и $\Omega_{u_{1}}^{v_{1}}=0$. Таким образом, доказано, что 1-формы $\omega_{u}^{v}$, входящие в равенства (73), удовлетворяют системе дифференциальных уравнений

$$
d \omega_{u}^{v}=\omega_{u}^{w} \wedge \omega_{w}^{v}
$$

Кроме того, в силу (73) имеем

$$
\sum_{u, v=0}^{n} \theta_{u v}^{u v}=2 \cdot \sum_{u, v=0}^{n} \omega_{(u}^{(u} \delta_{v)}^{v)}=n \cdot \sum_{u=0}^{n} \omega_{u}^{u}
$$


Поэтому из равенства (15) следует тождество

$$
\sum_{u=0}^{n} \omega_{u}^{u}=0 .
$$

Итак, 1-формы $\omega_{u}^{v}$, определенные формулами (72), удовлетворяют уравнениям Маурера-Картана групшы Ли $\mathrm{PGL}(n)$.

Перейдем к заключительному этапу доказательства. Для любой точки $x \in V$ существует ее окрестность $\bar{V}$, диффеоморфная некоторой области $M$ на $P^{n}$. Пусть $f_{n}^{2}: M \rightarrow P^{N}$ и $f: M \rightarrow P^{N}$ - отображения вложения такие, что: $f_{n}^{2}(M)=$ $\bar{V}_{n}^{2} \subset V_{n}^{2}$ - область на многообразии Веронезе, а $f(M)=\bar{V} \subset V$ - область на подмногообразии $V$. Тогда инвариантно определены два многообразия реперов $\mathscr{F}\left(\bar{V}_{n}^{2}\right) \subset \mathscr{F}\left(P^{N}\right)$ и $\mathscr{F}_{2}(\bar{V} \odot \bar{V}) \subset \mathscr{F}\left(P^{N}\right)$. При этом если $\hat{f}_{n}^{2}: \mathscr{F}\left(\bar{V}_{2}^{n}\right) \rightarrow \mathscr{F}\left(P^{N}\right)$ и $\hat{f}: \mathscr{F}_{2}(\bar{V} \odot \bar{V}) \rightarrow \mathscr{F}\left(P^{N}\right)$ - соответствующие вложения, то по доказанному

$$
\hat{f}^{*} \omega_{u_{1} u_{2}}^{v_{1} v_{2}}=\left(\hat{f}_{n}^{2}\right)^{*} \omega_{u_{1} u_{2}}^{v_{1} v_{2}}=2 \cdot \omega_{\left(u_{1}\right.}^{\left(v_{1}\right.} \delta_{\left.u_{2}\right)}^{\left.v_{2}\right)},
$$

где $\omega_{u_{1} u_{2}}^{v_{1} v_{2}}$ - формы Маурера-Картана группы Ли $\mathrm{PGL}(N)$, удовлетворяющие структурным уравнениям (5), (6), а $\omega_{u}^{v}$ - формы Маурера-Картанагруппы Ли $\mathrm{PGL}(n)$, удовлетворяющие структурным уравнениям (74), (75). Поэтому, применяя предложение 1 , можно заключить, что подмногообразия $\mathscr{F}_{2}(\bar{V} \odot \bar{V})$ и $\mathscr{F}\left(\bar{V}_{n}^{2}\right)$ совпадают с точностью до проективного преобразования пространства $P^{N}$, т.е.

$$
\mathscr{F}_{2}(\bar{V} \odot \bar{V})=g \cdot \mathscr{F}\left(\bar{V}_{n}^{2}\right),
$$

где $g \in \mathrm{PGL}(N)$. Так как левое действие группы $\mathrm{PGL}(N)$ на многообразии базисов $\mathscr{F}\left(P^{N}\right)$ и левое действие этой групшы на пространстве $P^{N}$ вместе с проекцией $\pi_{00}=p \circ e_{00}$ удовлетворяют тождеству $\pi_{00} \circ L_{g}=L_{g} \circ \pi_{00}$, то

$$
\bar{V}=\pi_{00}\left(\mathscr{F}_{2}(\bar{V} \odot \bar{V})\right)=\pi_{00}\left(g \cdot \mathscr{F}\left(\bar{V}_{n}^{2}\right)\right)=g \cdot\left(\pi_{00}\left(\mathscr{F}\left(\bar{V}_{n}^{2}\right)\right)\right)=g \cdot \bar{V}_{n}^{2},
$$

т.е. $\bar{V}=g \cdot \bar{V}_{n}^{2}$. Итак, подмногообразие $\bar{V}$ с точностью до проективного преобразования совпадает с областью $\bar{V}_{n}^{2}$ на многообразии Веронезе $V_{n}^{2}$. Таким образом, подмногообразие $V$ локально проективно эквивалентно многообразию Веронезе $V_{n}^{2}$.

Если теперь $V$ и $P^{n}$ диффеоморфны, то в качестве $M$ можно взять $P^{n}$. Но тогда $\bar{V}=V, \bar{V}_{n}^{2}=V_{n}^{2}$ и $V=g \cdot V_{n}^{2}$. Таким образом, подмногообразия $V$ и $V_{n}^{2}$ проективно эквивалентны. Теорема доказана.

СлеДСТвИЕ 1 . Пусть $V$ - гладкое $n$-мерное $(n \geqslant 2)$ подмногообразие в $P^{N}$ $(N=n(n+3) / 2)$, заданное векторным уравнением

$$
P=P(x)=P\left(x^{1}, x^{2}, \ldots, x^{n}\right)
$$

на некоторой области в $\mathbb{R}^{n}$. Это подмногообразие проективно эквивалентно области на многообразии Веронезе $V_{n}^{2}$ тогда и только тогда, когда для 
любьх $u=\left(u^{1}, u^{2}, \ldots, u^{n}\right) u v=\left(v^{1}, v^{2}, \ldots, v^{n}\right)$ из области определения функиии $P(x)$, где $u \neq v$, выполняются условия

$$
\begin{aligned}
& \left(\mathrm{A}^{\prime}\right) \operatorname{rank}\left\{P(u), \frac{\partial P(u)}{\partial x^{i}}, \frac{\partial P(v)}{\partial x^{j}}, P(v) \mid i, j=1, \ldots, n\right\}=2 n+1, \\
& \left(\mathrm{~B}^{\prime}\right) \operatorname{det}\left\{P(u), \frac{\partial P(u)}{\partial x^{i}}, \frac{\partial^{2} P(u)}{\partial x^{j} \partial x^{k}} \mid i, j, k=1, \ldots, n, \quad j \leqslant k\right\} \neq 0 .
\end{aligned}
$$

Действительно, в локальных координатах условия (А) и (В) теоремы имеют вид $\left(\mathrm{A}^{\prime}\right)$ и $\left(\mathrm{B}^{\prime}\right)$ соответственно.

Таким образом, практическое распознавание многообразий Веронезе сводится к вычислению определителей, составленных из производных не выше второго порядка. В частности, признак двумерного многообразия Веронезе $V_{2}^{2}$ в 5-мерном пространстве в локальных координатах записывается в виде

$$
\begin{aligned}
& \operatorname{rank}\left\{P(u), \frac{\partial P(u)}{\partial x^{1}}, \frac{\partial P(u)}{\partial x^{2}}, \frac{\partial P(v)}{\partial x^{1}}, \frac{\partial P(v)}{\partial x^{2}}, P(v)\right\}=5, \\
& \operatorname{det}\left\{P(u), \frac{\partial P(u)}{\partial x^{1}}, \frac{\partial P(u)}{\partial x^{2}}, \frac{\partial^{2} P(u)}{\partial x^{1} \partial x^{1}}, \frac{\partial^{2} P(u)}{\partial x^{1} \partial x^{2}}, \frac{\partial^{2} P(u)}{\partial x^{2} \partial x^{2}}\right\} \neq 0
\end{aligned}
$$

для любых $u$ и $v(v \neq u)$ из области определения векторной функции $P(x)$.

Доказательство теоремы легко может быть преобразовано в доказательство критерия принадлежности пары подмногообразий одному квадратичному многообразию Веронезе.

СЛЕДСТВИЕ 2. Пусть $V_{1} u V_{2}-$ пара гладких связныx $n$-мерных $(n \geqslant 2)$ многообразий, вложсенных в проективное пространство $P^{N} \quad(N=n(n+3) / 2)$ без пересечений и удовлетворяющих условиям:

(А) касательные проективные пространства для любой точки подмногообразия $V_{1}$ и любой точки подмногообразия $V_{2}$ пересекаются в точке;

(В) соприкасающиеся проективные пространства во всех точках подмногообразий $V_{1}$ и $V_{2}$ совпадают с обгемлющим пространством $P^{N}$.

Тогда для любой пары точек $A \in V_{1} u B \in V_{2}$ существуют их окрестности $\bar{V}_{1} \subset V_{1} u \bar{V}_{2} \subset V_{2}\left(A \in \bar{V}_{1}, \quad B \in \bar{V}_{2}\right)$ и существует проективное преобразование пространства $P^{N}$, переводящее подмногообразия $\bar{V}_{1} u \bar{V}_{2}$ в пару областей на многообразии Веронезе $V_{n}^{2}$.

Действительно, как и при доказательстве теоремы, условие (А) гарантирует, что с любой парой точек $P \in V_{1}$ и $Q \in V_{2}$ можно связать проективньй репер $\left\{A_{u v}\right\}$, определяемый условиями

$$
\begin{gathered}
P=A_{00}=p\left(e_{00}\right) \in V_{1}, \quad Q=A_{n n}=p\left(e_{n n}\right) \in V_{2}, \\
P T_{P}^{1}\left(V_{1}\right) \cap P T_{Q}^{1}\left(V_{2}\right)=A_{0 n}=p\left(e_{0 n}\right), \\
W_{P}^{1}\left(V_{1}\right)=\left\langle e_{00}, e_{01}, \ldots, e_{0 n}\right\rangle, \quad W_{Q}^{1}\left(V_{2}\right)=\left\langle e_{n n}, \ldots, e_{n 1}, e_{n 0}\right\rangle .
\end{gathered}
$$

Затем, рассматривая многообразие всех базисов $\mathscr{F}\left(V_{1} \times V_{2}\right)$ над парами точек $P \in$ $V_{1}$ и $Q \in V_{2}$, можно все рассуждения, приведенные в доказательстве теоремы, практически дословно перенести на данный случай. Разница будет состоять лишш в том, что теперь в качестве базы расслоения $\pi_{00} \times \pi_{n n}: \mathscr{F}\left(V_{1} \times V_{2}\right) \rightarrow V_{1} \times V_{2}$ и его редукций будет выступать прямое произведение $V_{1} \times V_{2}$ вместо $V \times V \backslash \operatorname{diag}(V \times V)$. 
СлЕДСТВИЕ 3. Пусть $V_{1} u V_{2}-$ пара гладких связных $n$-мерных $(n \geqslant 2)$ непересекающихся подмногообразий в $P^{N}(N=n(n+3) / 2)$, заданных векторными уравнениями

$$
P=P(x)=P\left(x^{1}, x^{2}, \ldots, x^{n}\right) \quad u \quad Q=Q(y)=P\left(y^{1}, y^{2}, \ldots, y^{n}\right) .
$$

Эти подмногообразия принадлежат одному многообразию Веронезе $V_{n}^{2}(c$ точностью до проективного преобразования пространства $\left.P^{N}\right)$ тогда и только тогда, когда для любого $u=\left(u^{1}, u^{2}, \ldots, u^{n}\right)$ из области определения функции $P$ и любого $v=\left(v^{1}, v^{2}, \ldots, v^{n}\right)$ из области определения функиии $Q$ выполняются условия

$$
\begin{aligned}
& \operatorname{rank}\left\{P(u), \frac{\partial P(u)}{\partial x^{i}}, \frac{\partial Q(v)}{\partial y^{j}}, Q(v) \mid i, j=1, \ldots, n\right\}=2 n+1, \\
& \operatorname{det}\left\{P(u), \frac{\partial P(u)}{\partial x^{i}}, \frac{\partial^{2} P(u)}{\partial x^{j} \partial x^{k}} \mid i, j, k=1, \ldots, n, \quad j \leqslant k\right\} \neq 0, \\
& \operatorname{det}\left\{Q(v), \frac{\partial Q(v)}{\partial y^{i}}, \frac{\partial^{2} Q(v)}{\partial y^{j} \partial y^{k}} \mid i, j, k=1, \ldots, n, \quad j \leqslant k\right\} \neq 0 .
\end{aligned}
$$

ЗАМЕЧАНИЕ 5 . Следствия 2 и 3 могут быть применены для любой конечной системы $n$-мерных подмногообразий $V_{1}, V_{2}, \ldots, V_{r}$ пространства $P^{N}$ при доказательстве их принадлежности одному многообразию Веронезе $V_{n}^{2}$.

В заключение автор выражает искреннюю благодарность В.. . Кириченко и Г. А. Клековкину за интерес к полученным результатам и их детальное обсуждение.

\section{Список литературы}

1. Akivis M. A., Goldberg V.V. Projective differential geometry of submanifolds. Amsterdam-London-New York-Tokyo: North-Holland, 1993.

2. Chern S. S., do Carmo M., Kobayashi S. Minimal submanifolds on the sphere with second fundamental form of constant length // Functional Analysis and Related Field (Proc. Conf. for M. Stone, Univ. Chicago, III, 1968). New York: Springer-Verlage, 1970. P. 59-75.

3. Griffiths $P$. On Cartan's method of Lie groups and moving frames as applied to uniqueness and existence questions in differential geometry // Duke Math. J. 1974. V. 41. № 4. P. $775-814$.

4. Griffiths P., Harris J. Algebraic geometry and local differential geometry // Ann. Sci. École Norm. Sup. 4 serie 1979. V. 12. P. $355-452$.

5. Little J.A., Pohl W.F. On tight immersions of maximal codimension // Invent. Math. 1971. V. 11. P. 179-204.

6. Nomizu K. A characterization of Veronese varieties // Nogoya Math. J. 1976. V. 60. P. $181-188$.

7. Nomizu K., Yano K. On circles and spheres in Riemannian geometry // Math. Ann. 1974. V. 210. P. 163-170.

8. Sasaki T. On the Veronese embedding and related system of differential equations // Global Differential Geometry and Global Analiysis. Lecture Note in Math. 1990. V. 1481. P. 210-293.

9. Коннов В. В. Дифференциальная геометрия некоторых классов алгебраических многообразий. Самара: СамГПУ, 1998. 